\title{
Heading Toward Trusted ATCO-AI Systems: A Literature Review
}

\author{
J. Y. Zhong, S. K. Goh, C. J. Woo \\ Air Traffic Management Research Insititute (ATMRI) \\ School of Mechanical and Aerospace Enginnering (MAE) \\ Nanyang Technological University (NTU), Singapore
}

[FOR SELF-REFERENCE ONLY. DO NOT DISTRIBUTE.]

\begin{abstract}
The current review addresses emerging issues that arise from the creation of safe, beneficial, and trusted artificial intelligenceair traffic controller (AI-ATCO) systems for air traffic management (ATM). These issues concern trust between the human user and automated or AI tools of interest, resilience, safety, and transparency. To tackle these issues, we advocate the development of practical AI-ATCO teaming frameworks by bringing together concepts and theories from neuroscience and explainable AI (XAI). By pooling together knowledge from both ATCO and AI perspectives, we seek to establish confidence in AI-enabled technologies for ATCOs. In this review, we present an overview of the extant studies that shed light on the research and development of trusted human-AI systems, and discuss the prospects of extending such works to building better trusted ATCO-AI systems. This paper contains three sections elucidating trust-related human performance, AI and explainable AI (XAI), and human-AI teaming.
\end{abstract}

\section{Index Terms}

Trusted ATCO-AI Systems, Neuroscience, Neuroimaging, Neuroscience-inspired AI, Machine Learning, Explainable AI (XAI), Human-AI Teaming

\section{INTRODUCTION}

Contemporary technology advances in automation, artificial intelligence (AI), and computational algorithms have created great benefits for human operators, as well as increases in adverse or unexpected consequences [1], [2], [3]. To ensure safe and seamless human-machine interaction (HMI), trust between humans and machines (in the form of AI or automated tools) has become a widely discussed topic over the past three decades [4], [5], [6]. In particular, within the air traffic management (ATM) domain, human-machine trust has long been recognized as essential for complex ATM systems to deliver the proposed capacity and safety benefits [7], [8].

Irrespective of the types of interactive agents involved, trust, in general terms, is a psychological state that can manifest itself as an intervening variable between particular external situations or experiences (e.g., social interactions) and human behaviors inherent to such situations [7]. More specific and operational definitions of human-machine trust that has been widely accepted in the current human factors literature concern the perception of trust as: (i) "the extent to which a user is willing to act on the basis of the information, recommendations, actions, and decisions of a computer-based tool or decision aid" (p. 11 in [7], adapted from p. 1 in [9])) or (ii) "the attitude that an agent will help achieve an individual's goals in a situation characterized by uncertainty and vulnerability" (p. 6 in [10]). Here, it is worth noting that according to the second operational definition, trust is characterized as decision-making heuristic that machine operators use in situations that are too uncertain or changing rapidly [3], [2].

Together with these two popular operational definitions of trust, the concept of calibrated trust must also be mentioned. Calibrated trust refers to the condition in which the anticipated or perceived trust of a machine, automation or AI matches the experienced trust associated with the actual use of the tool [10], [1], [3]. Seen in this light, trust becomes an evolving process that gets updated with increased information of the operating machine or system [1]. Following a decision-making framework, this means that a dynamic interaction between two variables occurs: (i) an initial perception or biased opinion of the machine or system before its actual use, (ii) updated perception and knowledge of the machine or system during and after usage [1], [11], [12].

With all these concepts of trust in mind, the direction of our current research aims to study the operational nature of trust and affiliated human factors (e.g., cognitive bias and constraints) in the ATM domain and apply such knowledge to the development of human-centered AI (HAI) systems for the purpose of achieving trusted human-AI teaming. As shown in Figure 1, our approach covers three primary components: (i) Human performance of air traffic controllers (ATCOs), (ii) HAI tools, and (iii) "human-in-the-loop" techniques and procedures, which incorporates trust as a major component. Currently, it is well known that ATCOs are using state-of-the-art technology to deliver safe and efficient air traffic services. A huge gap remains, however, in understanding "human-in-the-loop" processes and mechanisms tied to optimal cooperation between ATCOs and the automated or AI tools they are using [4]. Henceforth, in the sections below, we shall review recent works 
related to: (a) trust-related behavioral and neuroscience research in the HMI and ATM domains, (b) AI and Explainable (XAI) systems - and (c) discuss how knowledge gained from both human experimentation and AI development can be harnessed for building better trusted ATCO-AI systems.

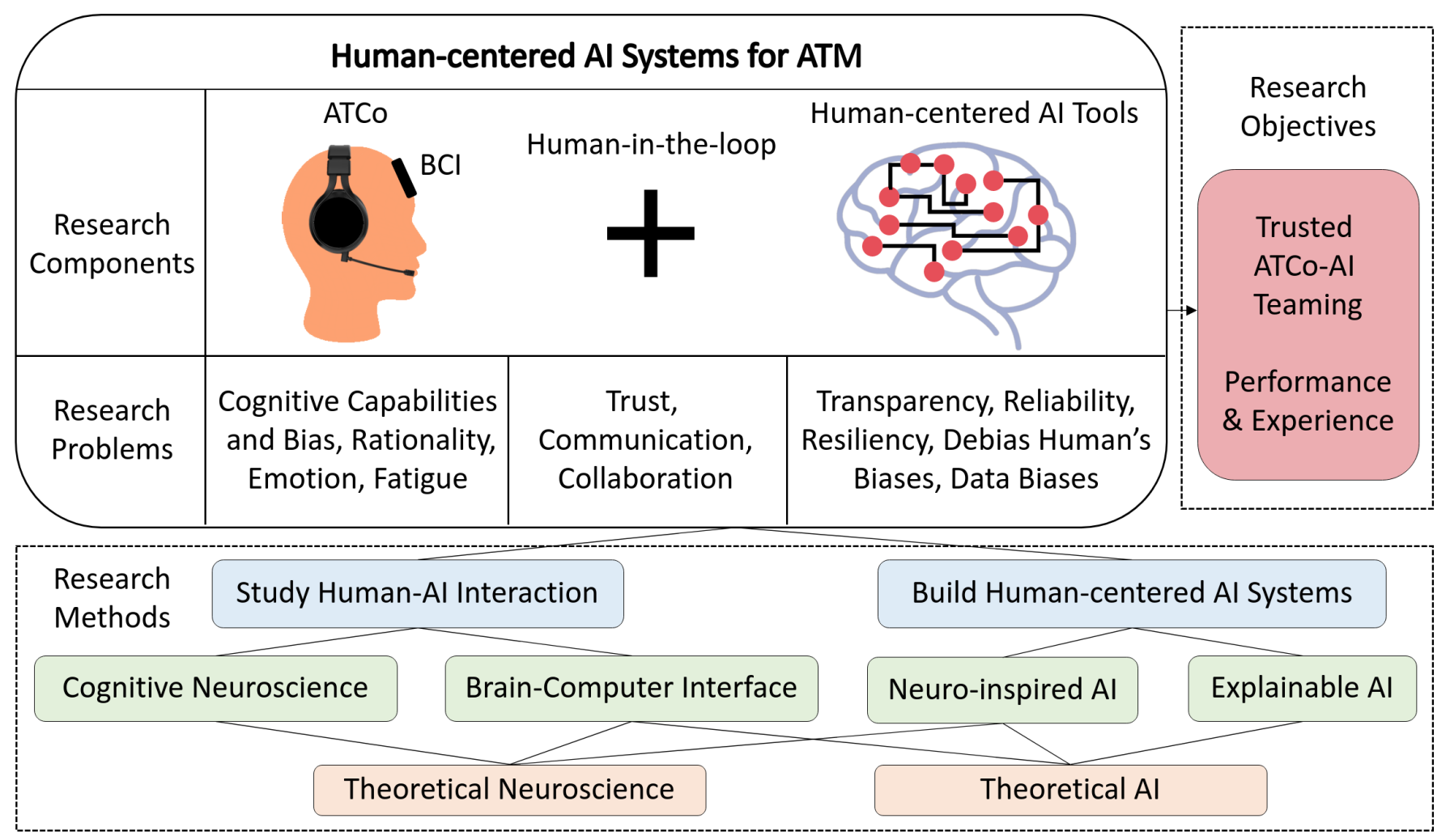

Figure 1: A proposed research framework for developing trusted HAI systems. COPYRIGHT CC BY-NC-ND 4.0, S. K. GOH \& J. Y. ZHONG, 2020, NANYANG TECHNOLOGICAL UNIVERSITY

\section{Trust-Related Human Performance}

Over the past three decades, numerous studies in the human factors and ergonomics domain had investigated trust between human users and automated tools, which is simply termed as human-automation trust [8], [7], [5], [6]. As AI tools and systems are currently undergoing rapid development and refinements in the ATM domain, we have yet to witness a published ATM study that made a systematic investigation of human-AI trust. Henceforth, this section will focus exclusively on human-automation trust in both ATM and non-ATM domains. Note that by "automation," we refer to the "machine execution of functions" (p. 286 [13]) and "technology that actively selects data, transforms information, makes decisions, or controls processes" (p. $50[10])$.

Trust has been conceptualized as an essential mechanism for automated ATM systems to deliver the proposed capacity and safety benefits [14]. However, research on trust in ATM systems has been scarce and and has almost all been done using questionnaires that gave a subjective measure of trust [8], [7], [5], [6]. For instance, a popular questionnaire that has been used widely in the ATM domain is the SHAPE Automation Trust Index (SATI) [15], which contains rating scales measuring ATCOs' overall level of trust and the constituents of trust such as reliability, predictability, and understandability. While SATI has been found to be easy to administer, it has been criticized for being unable to provide a multidimensional measure of trust [16]. This means that personality- and individual history-related aspects of trust cannot be assessed by SATI.

Apart from knowing that trust is commonly measured through self-report questionnaires, it is worth noting that a common trust-related issue in the use of automated ATM tools or systems relates to the phenomenon of complacency, which occurs when an ATCO places a high level of trust in an automated tool to the negligence or failure of monitoring the "raw" sources of information that provide inputs to the automated system [8], [5]. In the event of over-complacency or over-confidence in the functionalities of the automated system, system errors may go about undetected [17]. As for the measurement of complacency, the presence of complacency is usually inferred with respect to human performance data reflecting lower levels of attention and shorter monitoring when using automation [18], [19], [20]. For instance, operators have been shown to scan raw information sources less often when using automation than when performing the task manually and when automation reliability is higher 
rather than lower [19], [20]. In the same vein, when operators were given a tool that contains automated settings for extracting raw information sources, they used it much less often - indicative of faster data extraction - under automation than under manual control [18].

On another end, mistrust of an automated system, the polar opposite of complacency, can also pose significant risks for ATCO performance. Mistrust can either lead an ATCO to "over-monitor" the system in the event of errors [8] or ignore error warnings given by the system entirely [21]. The latter phenomenon has been dubbed the "cry wolf effect" [22] and has led to users of automated tools taking longer to to re-engage the use of automated services after experiencing system failures [23]. Moreover, mistrust is a negative psychological phenomenon that can persist even in the presence of a perfectly reliable automated tool [8]. For instance, using an automated conflict detection advisory in a simulated trajectory-based operation (TBO) airspace, Metzger and Parasumaran (2005) [19] showed that trust ratings of the automated tool were not high even though it was $100 \%$ reliable.

In addition to the aforementioned studies, current state-of-the-art studies of human-automation trust have incorporated neuroimaging technologies and techniques. Unlike questionnaires that provide subjective measures of trust, neuroimaging provide objective and real-time measurement of trust and trust-related psychophysiological signals emanating from the human brain and peripheral nervous system [24]. Specifically, these techniques encompass: (i) electroencephalogram (EEG), (ii) functional magnetic resonance imaging (fMRI), (iii) functional near-infrared spectroscopy (fNIRS), (iv) electrocardiogram (ECG), (v) electrodermal activity (EDA) recording, (vi) electromyography (EMG), (vii) electrooculography (EOG), (viii) eyetracking, and (ix) photoplethysmography (PPG) [25], [24]. Among these, the first three techniques relate to the recording of signals emanating from the brain reflective of changes in underlying neural activity while the latter five techniques involve the recording of signals from the peripheral or autonomic nervous system (i.e., from the heart-ECG, PPG; from the skin and musculature-EDA, EMG; from the eyes-EOG, eye-tracking).

In this paper, our main focus is on understanding the brain mechanisms of human-automation trust in ATM, and hence we shall only provide a review of notable brain imaging studies. In recognition of the fact that EEG has been the overwhelming favorite brain imaging technique used by researchers of human-automation trust and that we plan to harness fMRI for future projects on human-automation/AI trust in ATM, extant human-automation studies that utilized these two types of brain imaging techniques shall be the focus of this review. We elucidate the contributions of these two brain imaging technologies to humanautomation interaction and trust in the subsections below. Specifically, because we aimed at learning and adopting the newest state-of-the-art experimental methodologies in our future projects, we concentrated our efforts on a review of notable humanautomation trust studies utilizing EEG and fMRI studies over the five years only [2016 - 2021 (present)].

\section{A. EEG Studies}

\section{1) General Introduction}

EEG is a non-invasive, electrophysiological technique used for recording electrical signals emanating from brain activity. Multiple electrodes, either wet or dry, are placed on an individual's scalp to record the brain's spontaneous activity over a period of time. The instruments for conducting EEG are relatively inexpensive (compared with those for conducting fMRI and fNIRS) and highly portable. Unlike fMRI, which requires subjects to lie supine during the brain scan, subjects sit or stand in their normal upright position during EEG recording. Moreover, EEG offers higher temporal resolution than both fMRI and fNIRS, being able to record fluctuation in brain waves or rhythms on the scale of milliseconds [25]. Due to all these these factors, EEG has been the most preferred brain imaging technique for investigating neurocognitive mechanisms in the ATM domain [25].

\section{2) Mental Workload}

With respect to the cognitive factors investigated, a preponderance of ATM EEG studies focused on the cognitive factor of mental workload [25], [26]. In view of the prominence of such studies and the practical implications they convey, notable findings and concepts from these studies shall be presented first before discussing trust-related EEG studies. In layman's terms, mental workload can be conceived as the amount of information processing an ATCO has to perform in response to the operational demands of the system(s) under his/her control [27]. EEG power spectrum analysis, used to identify changes in the EEG spectra reflecting different levels of mental workload, has consistently shown the correlation of certain EEG spectra with task difficulty or complexity. Most notably, numerous studies have shown that an increase of the theta band spectral power $(4-7 \mathrm{~Hz})$ over the frontal cortex and a decrease in the alpha band spectral power $(8-12 \mathrm{~Hz})$ over the parietal cortex correlated positively with an increase in mental workload or task complexity [28], [29], [30], [31]. For instance, Borghini et al. (2017) [29], through the use of machine learning-based automatic stop stepwise linear discriminant analysis (asSWLDA) of EEG spectral features, showed that frontal theta power spectral density (PSD) increased with ATCOs' operational task complexity while frontal alpha PSD decreased more when automatic actions are performed (e.g., opening up a pie menu to select an option for an aircraft) than when more intentional or deliberative activities were performed (e.g., when conflicts and flight plan deviations were spotted). In contrast to the changes in the frontal alpha PSD, theta PSD in the parietal cortex increased more when conscious planning and decision making were called for than when effortless or automatic actions were performed. With reference to a skill, rule, and knowledge (SRK) model, increases in frontal alpha, frontal theta, and parietal 
theta PSDs were proposed to reflect rule-based and knowledge-based behaviors, with the latter requiring a higher degree of synchronization of all three types of EEG rhythms than the former (Figure I). Specifically, rule-based behavior relate to following a learned rule or procedure during problem solving or planning while knowledge-based behavior is more cognitively demanding, requiring a person to engage in stored and newly acquired memories or knowledge in order to reach a desired goal. On the other hand, a decrease in frontal alpha PSD (or alpha desynchronization) was proposed to reflect skill-based behavior, automatic actions that occur without mental exertion.

Table I: Hypothesized EEG rhythms assigned to the cognitive control behaviors of skill, rule, and knowledge (SRK). The SRK behaviours were described in terms of variation from a reference condition: synch — synchronization; desynch desynchronization. [Source: Table 1, Borghini et al. (2017)]

\begin{tabular}{|l|l|l|l|}
\hline \multirow{2}{*}{ COGNITIVE CONTROL BEHAVIOUR } & \multicolumn{2}{|l|}{ EEG RHYTHM } & \multicolumn{2}{l|}{} \\
\cline { 2 - 4 } & Frontal Theta & Parietal Theta & Frontal Alpha \\
\hline Skill & Lowest Synch & Lowest Synch & De-synch \\
\hline Rule & Synch & Synch & Synch \\
\hline Knowledge & Highest Synch & Highest Synch & Synch \\
\hline
\end{tabular}

Due to the fact that workload can be influenced by other cognitive factors such as attention, vigilance, mental fatigue, and environmental factors such as operational task demands and complexity, the establishment of valid and reliable methods for measuring mental workload is of top priority for human factors researchers in ATM [25]. Regarding this, numerous recent ATM studies have employed machine learning algorithms and models such as linear discriminant analysis (LDA) and support vector machine (SVM) to classify EEG-based spectral features (obtained from ATCO participants) into different categories of workload during real-time air traffic simulations [32], [33], [29], [34]. These EEG-based workload indices provide objective and rapid online assessment of task engagement and perceived workload on the scale of seconds or minutes, and can be readily applied to trigger adaptive automation (AA) solutions for brain-computer interface (BCI) systems [33], [25]. For instance, Aricò et al. (2016) [33] computed a real-time EEG-based workload index through asSWLDA of frontal theta and parietal alpha bands. When this workload index was classified online as high, AA solutions were triggered (e.g., highlighting a calling aircraft on the radar to facilitate an ATCO's pinpointing of its location). By contrast, no AA solutions were triggered when the workload index was classified as low. By triggering AA solutions in response to the online workload status of the ATCO in this way, a reduction in ATCOs' workload co-occurred with an enhancement in operational performance (i.e., faster responses to operational demands), particularly under simulated air traffic scenarios with high task difficulty. In addition, a recent EEG study by Radüntz et al. (2020) [34] showed that an a workload index computed through Dual Frequency Head Maps (DFHM) method - which involved a SVM-based classification algorithm - increased in a stepwise fashion as the number of aircraft to be handled by each ATCO per hour increased. Interestingly, relatively high correlations were observed between this objective workload measure and subjective, questionnaire-based reports of workload $(.67 \leq r \leq .81)$ under scenarios with equal volumes of air traffic.

3) Trust

Over the past five years, there has only been a limited set of EEG studies that investigated human-related trust. We found six noteworthy EEG studies and tabulated their key features - experimental task(s) and design, data analysis technique(s), main findings and implications - in Table II below. A phenomenon common to all these studies pertains to the use of experimental design or tasks that involved the modulation of reliability (or dependability) offered by an automated tool (Table II, column 2). Reliability refers to how consistent an automation can perform in providing accurate information and was largely manipulated through the programming and presentation of automated agents or advisories that can provide information with different probabilities of accuracy [35], [1], [36], [3] or risk-taking tendencies [37], [38].

More importantly, with respect to the brain regions that were activated during human-automation trust-related decision making, these recent EEG studies pinpointed anterior regions such as the (i) lateral prefrontal cortex [38], (ii) anterior cingulate cortex (ACC) [1], [3], and posterior regions such as the (iii) occipital cortex [38] and (iv) fusiform gyrus (also known as the occipitotemporal gyrus) [3]. Two studies further showed that power increases or variation in the beta frequency band (12 $\mathrm{Hz}-35 \mathrm{~Hz}$ ) were associated with increased levels of trust [36] and making discriminatory judgments between trustworthy and untrustworthy stimuli [35]. Figure 2 shows the brain regions from which trust-related decision making EEG signals were recorded. Figure 2A shows the effective connectivity network uncovered by Sanders et al. (2019) [3] during a participant's 
detection of mistakes made by the automated advisory while Figure 2B shows the locations of the EEG electrodes, mapped out by Wang et al. (2018) [38], at which significant trust-related activations were found.

\section{B. fMRI Studies}

\section{1) General Introduction}

Functional Magnetic Resonance Imaging (fMRI) began in the early 1990s with its initial development generally credited to Seiji Ogawa and Ken Kwong [39]. Today, it is regarded as one of the most well-known neuroimaging techniques. fMRI works by inferring brain activity from changes in the blood flow in the brain. Changes in cerebral blood flow translated to changes in the magnetization levels of oxygenated and deoxygenated blood. A higher inflow of oxygenated blood into a particular brain region usually occurs when a person is thinking and performing a particular task, and this will lead to a rise in the magnetic signal that surrounds the cerebral capillaries in that particular region. This signal is recorded by the fMRI scanner and is called as "blood-oxygen-level-dependent (BOLD)", meaning that there is a coupling between cerebral blood flow into a brain region and the neuronal activation in that region [40]. In other words, when an area of brain is "activated" by some mental processes, the flow of oxygenated blood into it increases.

Due to the application of blood flow and oxygen metabolism to infer brain activity, fMRI offers several significant capabilities. It is non-invasive, does not involve radiation, and is generally safe for most people. It offers good spatial resolution of the brain to an accuracy of up to $1.0 \mathrm{~mm}$ and temporal resolution of up to 1.0 second [40]. It renders structural or anatomical images of the brain, as well its functional images, which represent hemodynamic responses to decisions and/or responses made over the period of the brain scan [40]. Based on statistical analyses that commonly operate on the basis of General Linear Models, functional brain images are normally always color-coded to convey the regions of significant brain activity [41]. This makes it easy for any reader to discern the location of different brain regions.

2) Trust

Over the past five years, there has only been three notable studies on human-automation trust [42], [43], [44]. Two investigated brain activations and effective connectivity between regions of interest through the use of a X-ray luggage screening task that presented automated advisories with varying proportion of false alarms [42] and misses [43]. [Note that a false alarm refer an emergency alarm (or alert) that is activated under an situation that does not require it [42], [7] while a miss refer to the absence of an alarm under an emergency situation that truly requires it [43].] The remaining and more recent study was a proof-of-concept study that investigated human-automation trust in the form of a quantum-inspired mathematical model [44] - to date, it was the only published trust-related study in the ATM domain.

Table III lists the experimental tasks and design, data analysis techniques, main findings and implications tied to these three studies. From a quick overview of the brain-related findings, several facts are worth highlighting: (i) Even though the behavioral response associated with advice utilization can be the same for both human and machine agents, the brain activation patterns in response to the advice given by these agents can be different [43]. (ii) Greater neural resources, as shown through brain activations observed from contrast analyses, during decision making in response to advice given by a human agent than to advice given by an automated agent - and these activation patterns differ based on the proportion of false alarms (Figures 3 $\mathrm{A}$ and B) and misses (Figures. $3 \mathrm{C}$ and D) experienced by the user [42], [43]. (iii) Human-to-machine-agent contrasts done by Goodyear et al. $(2016,2017)$ [42], [43] showed activations in the ACC and insula; the same findings were uncovered by Pushparaj et al. (2019) [44], who postulated ACC activation to reflect reciprocal trust and insular activation to reflect pure distrust. (iv) ACC activation has also been shown in human-human (interpersonal) trust under using an voluntary trust game that require mutual trust between two participants in deciding the amount of financial payoffs [45]. This shows that the ACC is vital for representing or inferring the mental states of another agent, irrespective of whether it is a human or an automated tool.

With these facts in mind, it is worth mentioning that there is current movement toward the treatment of human-automation trust as a decision-making process [2]. Specifically, this process frames trust in the form of value-based decisions that required the human user to continuously weigh the expected personal value of letting the automation to complete the task versus performing it manually [2], [46], [47]. In this way, any trust-related assessment must be made against a backdrop of risk and/or reward that may come with human-automation cooperation. Through a critical review of the decision neuroscience literature, Drnec et al. (2016) [2] identified an assortment of brain regions, located in the prefrontal, parietal, and limbic regions that can be involved in trust-related value-based decisions. As shown in Table IV, these hypothesized value-based regions involve the amygdala, ventral striatum, and ventral medial prefrontal cortex (vmPFC). Currently, to our knowledge, it is unknown as to: (i) how many of these regions will be activated during human-automation trust-related decision making, (ii) the extent to which they are activated and the scale of functional connectivity between them, and (iii) how these different brain activation and functional connectivity patterns change over time - under different physical or social environments. These issues show that there are many unknowns concerning the neural correlates of human-automation trust and that further investigations are definitely needed. 
Table II: Summary of Key EEG Studies of Human-Automation Trust done over the past Five Years (2016-2020)

\begin{tabular}{|c|c|c|c|}
\hline $\begin{array}{l}\text { Authors (Year } \\
\text { of publication) }\end{array}$ & $\begin{array}{c}\text { Experimental Task(s) and } \\
\text { Design }\end{array}$ & $\begin{array}{l}\text { Data Analysis } \\
\text { Technique(s) }\end{array}$ & Trust-relevant Findings and Implications \\
\hline $\begin{array}{l}\text { Oh, Seong, Yi, } \\
\text { \& Park (2020) }\end{array}$ & $\begin{array}{l}\text { 2D racing game with varying } \\
\text { levels of automated control. } \\
\text { Participants' self-reported } \\
\text { trust ratings were strongly } \\
\text { associated with the levels of } \\
\text { automated control provided. } \\
\text { Higher trusted emanated } \\
\text { from higher automated } \\
\text { control. }\end{array}$ & $\begin{array}{l}\text { Power spectral } \\
\text { density (PSD) } \\
\text { analysis }\end{array}$ & $\begin{array}{l}\text { Power of alpha and beta waves increased during } \\
\text { trials with high levels of trust ( }>90 \%) \text {. Power of } \\
\text { gamma waves increased during trials with low levels } \\
\text { of trust }(\leq 30 \%) \text {. Observed power increases are } \\
\text { collected from electrodes over the entire scalp. } \\
\text { Regions of interest were not reported. } \\
\text { Implication: Alpha waves are related to meditation } \\
\text { and anxiety reduction. Beta waves are associated } \\
\text { with conscious, attention-demanding thinking } \\
\text { processes. Gamma waves are related with anxiety } \\
\text { and higher-order information processing. }\end{array}$ \\
\hline $\begin{array}{l}\text { Jung, Dong, \& } \\
\text { Lee (2019) }\end{array}$ & $\begin{array}{l}\text { Decision-making task with } \\
\text { human- and machine-face } \\
\text { agents with different risk- } \\
\text { taking personalities. Risk- } \\
\text { taking personality of each } \\
\text { machine agent was } \\
\text { computed using an equation } \\
\text { that factored in reward size, } \\
\text { probability of correct } \\
\text { response, and risk-taking. }\end{array}$ & $\begin{array}{l}\text { Time-frequency } \\
\text { analysis } \\
\text { EEG power } \\
\text { variation analysis }\end{array}$ & $\begin{array}{l}\text { EEG average power variation is higher for both } \\
\text { agent's correct (AC) and wrong (AW) decisions when } \\
\text { the agent had a human face compared to when it } \\
\text { had a machine (robot) face. This pattern of results } \\
\text { held in both high and low trust conditions. } \\
\text { Implication: Participants' neural responses were } \\
\text { enhanced by an agent's external human-likenesses. } \\
\text { Participants were less sensitive to the participation } \\
\text { of less trusted agents if they were externally less } \\
\text { human-like. }\end{array}$ \\
\hline $\begin{array}{l}\text { Sanders, } \\
\text { Choo, ... Fitts } \\
(2019)\end{array}$ & $\begin{array}{l}\text { Multi-Attribute Test Battery } \\
\text { (MATB) of the US Air Force } \\
\text { In the MATB, participants } \\
\text { monitored the extent to } \\
\text { which four needles of a } \\
\text { gauge fell within a nominally } \\
\text { acceptable range. Four types } \\
\text { of 'algorithms,' varying } \\
\text { across two levels of } \\
\text { credibility (novice, expert) } \\
\text { and two levels of reliability } \\
\text { (low - 60\\
%, high - } 80 \text { \%) } \\
\text { were presented to } \\
\text { participants based on a } 2 \times 2 \\
\text { design over many trials. } \\
\text { Experimental design adapted } \\
\text { from that used by de Visser } \\
\text { et al. (2018) [see row below]. }\end{array}$ & $\begin{array}{l}\text { Effective } \\
\text { connectivity } \\
\text { analysis (ECA), } \\
\text { which shows the } \\
\text { causal flow of } \\
\text { dynamic time- } \\
\text { frequency EEG } \\
\text { data, were } \\
\text { performed on } \\
\text { independent } \\
\text { components (also } \\
\text { known as } \\
\text { maximally } \\
\text { independent time } \\
\text { source series) } \\
\text { located in the } \\
\text { fusiform gyrus, } \\
\text { cingulate cortex, } \\
\text { and prefrontal } \\
\text { cortex. }\end{array}$ & $\begin{array}{l}\text { Strong flow of time-frequency information from the } \\
\text { source node of fusiform gyrus (FG) [source node] to } \\
\text { the dorsal anterior cingulate cortex (dACC) and } \\
\text { posterior cingulate cortex (dPCC) [terminal nodes] } \\
\text { when participants observed the automation to be } \\
\text { working successfully under the high reliability } \\
\text { condition. } \\
\text { Implication: ECA findings supported extant notions } \\
\text { of the ACC and the PCC as the critical sites for } \\
\text { conflict monitoring and error detection and } \\
\text { suggested them to be important for calibrating one's } \\
\text { trust to the perceived trustworthiness of an } \\
\text { automated device. }\end{array}$ \\
\hline $\begin{array}{l}\text { de Visser, } \\
\text { Beatty, ... } \\
\text { McDonald } \\
\text { (2018) }\end{array}$ & $\begin{array}{l}\text { Flanker task: Popular task } \\
\text { used for measuring attention } \\
\text { and executive functioning. } \\
\text { Participants viewed how a } \\
\text { Flanker task was performed } \\
\text { based on pre-configured } \\
\text { 'algorithms' with low and } \\
\text { high degrees of reliability. }\end{array}$ & $\begin{array}{l}\text { Event-related } \\
\text { potential (ERP) } \\
\text { component } \\
\text { analysis }\end{array}$ & $\begin{array}{l}\text { Observational error positivity (oPe), an event-related } \\
\text { potential (ERP) arising putatively from the anterior } \\
\text { cingulate cortex (ACC), was detected in participants' } \\
\text { frontocentral scalp region when they detected } \\
\text { erroneous responses made by the 'algorithms.' The } \\
\text { amplitude of oPe was higher in the presence of error } \\
\text { responses than in the presence of correct responses } \\
\text { and this power difference was larger when observing } \\
\text { errors made by a high reliability 'algorithm' than } \\
\text { when observing errors made by a low reliability } \\
\text { 'algorithm.' Interestingly, oPe correlated with } \\
\text { questionnaire-based ratings of human-automation } \\
\text { trust to a relatively high degree ( } R^{2}=.65 \text { ). } \\
\text { Implication: The presence of oPe probably } \\
\text { represented a conscious orientation of attention } \\
\text { toward unexpected events (i.e., error responses in }\end{array}$ \\
\hline
\end{tabular}




\begin{tabular}{|c|c|c|c|}
\hline & & & $\begin{array}{l}\text { the high reliability 'algorithm's' case). With respect } \\
\text { to the correlational findings, this form of conscious } \\
\text { awareness could be modulated by trust-related } \\
\text { mechanisms. }\end{array}$ \\
\hline $\begin{array}{l}\text { Wang, } \\
\text { Hussein, ... } \\
\text { Abbass (2018) }\end{array}$ & $\begin{array}{l}\text { Trustworthiness task with } \\
\text { three types of artificial } \\
\text { agents: (i) low profit/risk- } \\
\text { taking, (ii) medium } \\
\text { profit/risk-taking, (iii) high } \\
\text { profit/risk-taking. } \\
8 \text { scenarios, each with } 30 \\
\text { rounds/trials. Number of } \\
\text { trustworthy agents varied } \\
\text { from } 0 \text { to } 3 \text { across the } \\
\text { scenarios. }\end{array}$ & $\begin{array}{l}\text { Autoregressive } \\
\text { modelling of time- } \\
\text { dependent EEG } \\
\text { dynamics, } \\
\text { estimation of EEG } \\
\text { complexity using } \\
\text { the entropy } \\
\text { measure, power } \\
\text { spectrum analysis } \\
\text { using discrete } \\
\text { Fourier transform. }\end{array}$ & $\begin{array}{l}\text { Participants showed higher neural responses in the } \\
\text { frontal (left, fronto-central, right) and occipital } \\
\text { cortices with higher perception of trustworthiness of } \\
\text { the artificial agent. } \\
\text { Implication: Frontal activations in general reflected } \\
\text { executive functioning and decision-making. Fronto- } \\
\text { central activation potentially reflected conflict } \\
\text { monitoring, error detection, and decision making } \\
\text { about risk and reward. Occipital lobe reflected } \\
\text { processing of visual information. }\end{array}$ \\
\hline $\begin{array}{l}\text { Akash, Hu, } \\
\text { Jain, \& Reid } \\
\text { (2018) }\end{array}$ & $\begin{array}{l}\text { Obstacle detection task. An } \\
\text { automated road obstacle } \\
\text { sensor was programmed } \\
\text { with } 50 \% \text { reliability on half of } \\
\text { the experimental trials and } \\
100 \% \text { reliability on the other } \\
\text { half. Brain signals related to } \\
\text { trust were collected from the } \\
100 \% \text { reliability trials while } \\
\text { brain signals related to } \\
\text { distrust were collected from } \\
\text { the } 50 \% \text { reliability trials. }\end{array}$ & $\begin{array}{l}\text { Machine learning } \\
\text { (ML)-based } \\
\text { modelling } \\
\text { classification of } \\
\text { EEG and galvanic } \\
\text { skin response } \\
\text { features. }\end{array}$ & $\begin{array}{l}\text { Two types of "trust-sensor models" (one general and } \\
\text { one customized) were created using a common set } \\
\text { of EEG and GSR features derived from the entire } \\
\text { sample of participants and a smaller set of EEG and } \\
\text { GSR features derived from comparisons of significant } \\
\text { features between each participant. feature } \\
\text { selection. EEG mean frequencies and power } \\
\text { variation over the frontocentral region of scalp } \\
\text { contributed to the validation of the trust-sensor } \\
\text { models. Power variation in the beta band found over } \\
\text { the parietal cortex (posterior scalp region) was } \\
\text { largely observed in the contrast between reliable } \\
\text { and unreliable advisories, suggesting that parietal } \\
\text { beta waves were involved in the processing of trust- } \\
\text { related perceptual information. }\end{array}$ \\
\hline
\end{tabular}

Note. Names of brain regions were bolded. 
A.

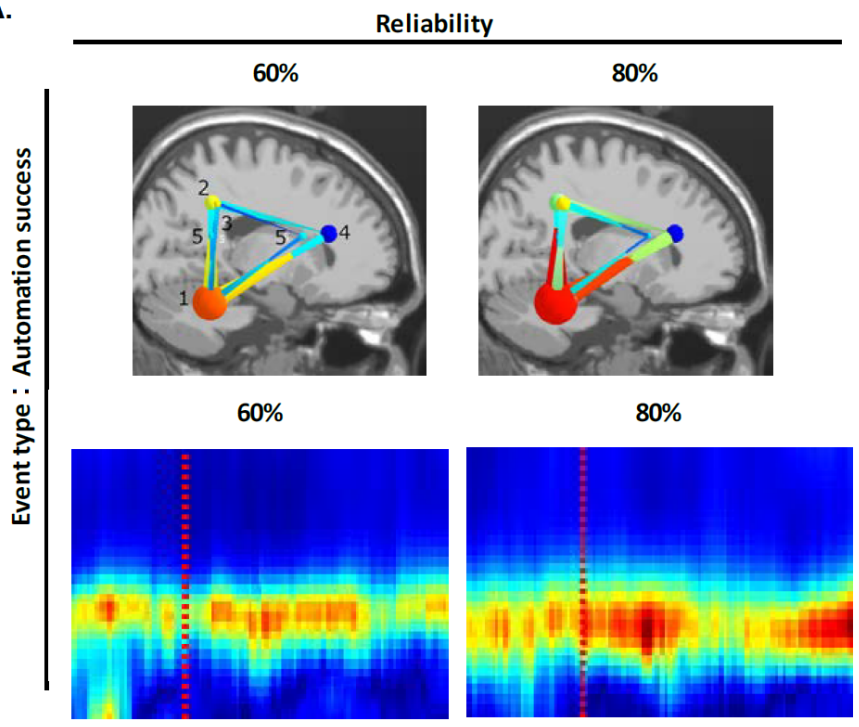

B.

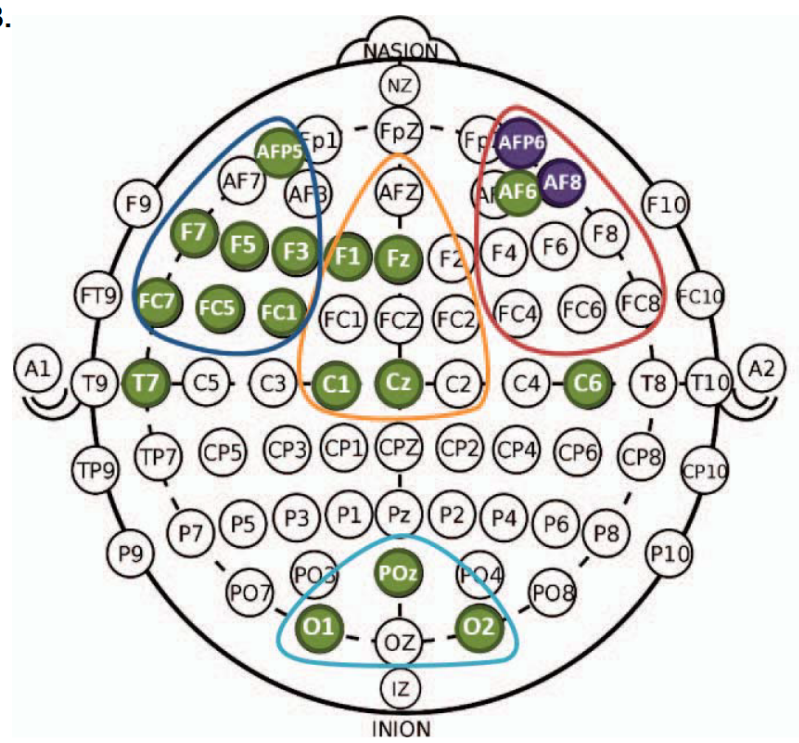

Figure 2: (A) EEG-based effective connectivity findings taken from one participant. There was strong flow of time-frequency information (as indicated by the bright red color) from the source node of fusiform gyrus (FG) [source node] to the dorsal anterior cingulate cortex (dACC) and posterior cingulate cortex ( $\mathrm{dPCC}$ ) [terminal nodes] when the automation was observed to be working successfully under the high reliability condition. Lighter colors in the low reliability condition indicate relatively weaker flow of time-frequency information. These findings were time-locked to the occurrence of the observational error positivity (oPE, see Table 1, row no. 4) 200 milliseconds after the onset of the automated event. [Source: Figure 3, Sanders et al. (2019) [3]] (B) Map of EEG electrodes that recorded significant human-automation trust-related signals from the frontal and occipital cortices. [Source: Figure 5, Wang et al., 2018 [38]] 
Table III: Summary of Key fMRI Studies of Human-Automation Trust done over the past Five Years (2016-2019)

\begin{tabular}{|c|c|c|c|}
\hline $\begin{array}{l}\text { Authors (Year } \\
\text { of Publication) }\end{array}$ & $\begin{array}{c}\text { Experimental Task(s) and } \\
\text { Design }\end{array}$ & $\begin{array}{c}\text { Neuroimaging } \\
\text { Analysis } \\
\text { Technique(s) }\end{array}$ & Trust-relevant Findings and Implications \\
\hline $\begin{array}{l}\text { Pushparaj, } \\
\text { Ayeni, ... Duong } \\
\text { (2019) }\end{array}$ & $\begin{array}{l}\text { Air Traffic Conflict } \\
\text { Detection Task. } \\
\text { Participants viewed five } \\
\text { conflict scenarios and } \\
\text { decided whether to accept } \\
\text { or reject the advice given } \\
\text { by an automated advisory } \\
\text { tool that presents prompt } \\
\text { advisory (text messages). } \\
\text { A quantum-inspired } \\
\text { mathematical model was } \\
\text { used to define trust. Trust } \\
\text { was defined as pure trust, } \\
\text { pure distrust, reciprocal } \\
\text { trust, and reciprocal } \\
\text { distrust. } \\
\text { Pure trust and pure } \\
\text { distrust reciprocal were } \\
\text { conceptualized as } \\
\text { independent constructs } \\
\text { while reciprocal trust and } \\
\text { distrust were } \\
\text { conceptualized as co- } \\
\text { dependent constructs. } \\
\text { Based on the model } \\
\text { conceived, the } \\
\text { aforementioned states of } \\
\text { trust can be superimposed } \\
\text { in accordance to the } \\
\text { principle of superposition. }\end{array}$ & $\begin{array}{l}\text { Seed-based } \\
\text { correlation } \\
\text { analysis (SCA). } \\
\text { SCA explores } \\
\text { the functional } \\
\text { connectivity } \\
\text { brain regions } \\
\text { based on } \\
\text { correlating the } \\
\text { time series of } \\
\text { the relevant } \\
\text { seed voxels of } \\
\text { activation. }\end{array}$ & $\begin{array}{l}\text { When using insula as the seed, insular activation was } \\
\text { found to correlate significantly with activations in the } \\
\text { insula, anterior cingulate cortex (ACC), and putamen } \\
\text { during the third conflict scenario, suggesting a } \\
\text { simultaneous activation of pure distrust, reciprocal } \\
\text { trust/distrust, and pure trust, respectively. } \\
\text { When using ACC as the seed, ACC activation was found to } \\
\text { correlate with the activations in the insula, and putamen } \\
\text { during the second prompt and the fifth conflict scenario, } \\
\text { suggesting a simultaneous activation of reciprocal } \\
\text { trust/distrust, pure distrust, and pure trust, respectively. } \\
\text { Implication: The fMRI findings provided the first findings } \\
\text { for validating the quantum-inspired trust model. The } \\
\text { main findings of simultaneous activations of two or more } \\
\text { brain regions associated with the different states of trust } \\
\text { suggested that mental representations of different trust } \\
\text { states can be superimposed upon each other when } \\
\text { monitoring air traffic and prior to deciding on the } \\
\text { acceptance of an automated advisory. }\end{array}$ \\
\hline $\begin{array}{l}\text { Goodyear, } \\
\text { Parasuraman, ... } \\
\text { Krueger (2017) }\end{array}$ & $\begin{array}{l}\text { Same X-ray screening task } \\
\text { as used by Goodyear et al. } \\
\text { (2016), see row below) } \\
\text { Bad advice refers to } \\
\text { "misses" (knife = present, } \\
\text { advice = absent) rather } \\
\text { than "false alarms (knife = } \\
\text { present, advice = absent)." } \\
\text { Good advice: Hits (knife = } \\
\text { present, advice = present; } \\
60 \% \text { of trials) } \\
\text { Bad advice: Misses ( } 40 \% \text { of } \\
\text { trials) } \\
\text { Same three-way mixed } \\
\text { model factorial design as } \\
\text { used by Goodyear et al. } \\
\text { (2016). }\end{array}$ & $\begin{array}{l}\text { Same analyses } \\
\text { as used by } \\
\text { Goodyear et al. } \\
\text { (2016) }\end{array}$ & $\begin{array}{l}\text { Behavioral findings were largely consistent with previous } \\
\text { findings shown by Goodyear et al. (2016). Main effects of } \\
\text { trust and advice utilization percentage were found. } \\
\text { Participants gave lower trust ratings after the fMRI scan } \\
\text { than before the fMRI scan. Advice utilization lower in the } \\
\text { presence of bad advice than in the presence of good } \\
\text { advice. Unlike previous findings by Goodyear et al. } \\
\text { (2016), no main or interactive effects of agent were } \\
\text { found. } \\
\text { During the decision phase (to search or to clear the } \\
\text { misses" (passage of the luggage), brain activations } \\
\text { (human > machine agent) were found in: } \\
\text { left anterior precuneus (L. aPreC) } \\
\text { right lingual gyrus (R. LG) } \\
\text { left cuneus (L. CUN) } \\
\text { During the feedback phase (slide showing whether a } \\
\text { knife was present or absent), brain activations } \\
\text { (machine > human agent) were found in: } \\
\text { right precentral gyrus (R. PrG) } \\
\text { right inferior parietal lobule (R. IPL) } \\
\text { left putamen (L. Pu) }\end{array}$ \\
\hline
\end{tabular}




\begin{tabular}{|c|c|c|c|}
\hline & & & $\begin{array}{l}\text { Granger causality analysis (GCA) showed that during the } \\
\text { decision phase, R. LG was the source of a brain network } \\
\text { with output connections to target ROIs R. anterior } \\
\text { cingulate cortex (R. ACC), L. PreC, and L. CUN. During the } \\
\text { feedback phase, left fusiform gyrus (L. FG) was found to } \\
\text { send out output connections to R. IPL. } \\
\text { Implication: Replicated previous findings by Goodyear et } \\
\text { al. (2016) in showing that greater neural resources were } \\
\text { consumed during decision-making in response to advice } \\
\text { given by a human agent than to advice given by a } \\
\text { machine agent. Effective connectivity findings derived } \\
\text { from GCA suggested that the LG modulated visual } \\
\text { attention in this decision-making process through a } \\
\text { bottom-up processing of task-relevant information. }\end{array}$ \\
\hline $\begin{array}{l}\text { Goodyear, } \\
\text { Parasuraman, ... } \\
\text { Krueger (2016) }\end{array}$ & $\begin{array}{l}\text { X-ray screening task } \\
\text { involving consideration } \\
\text { and acceptance of advice } \\
\text { from human and machine } \\
\text { agents. Advice pertained } \\
\text { to whether to search or to } \\
\text { clear the passage of a } \\
\text { luggage for the presence } \\
\text { of a knife. } \\
\text { Good advice: Hits (knife = } \\
\text { present, } 50 \% \text { of trials) } \\
\text { Bad advice: False alarms } \\
\text { (knife = present, advice = } \\
\text { absent; } 40 \% \text { of trials) } \\
\text { Three-way mixed model } \\
\text { factorial design. Between- } \\
\text { subjects IV: } 1 \text {. Advice } \\
\text { (human vs. machine). } \\
\text { Within-subjects IV: } 1 . \\
\text { Advice (good vs. bad), } 2 \text {. } \\
\text { Time (fMRI run1 vs. run2) }\end{array}$ & $\begin{array}{l}\text { Between- } \\
\text { subjects } \\
\text { contrast } \\
\text { analysis. } \\
\text { Effective } \\
\text { Connectivity } \\
\text { Analysis via } \\
\text { multivariate } \\
\text { Granger } \\
\text { causality } \\
\text { analysis (GCA) }\end{array}$ & $\begin{array}{l}\text { Self-reported trust in the human agent, not the machine } \\
\text { agent, was lower post-scan when compared with pre- } \\
\text { scan. Advice utilization percentage was lower in run } 2 \\
\text { under the bad advice condition in the presence of the } \\
\text { human agent compared with the machine agent. } \\
\text { During the decision phase (to search or to clear the } \\
\text { Parasuraman passage of the luggage), brain activations } \\
\text { (human > machine agent) found in (Table 1, p. 9): } \\
\text { right posterior insula (R. PI) } \\
\text { right anterior precuneus (R. aPreC) } \\
\text { left aPreC } \\
\text { left posterior cingulate cortex (L. PCC) } \\
\text { left rostrolateral prefrontal cortex (L. rIPFC) } \\
\text { During the feedback phase (slide showing whether a } \\
\text { knife was present or absent), brain activation (good } \\
\text { advice > bad advice) in the left dorsomedial prefrontal } \\
\text { cortex (L. dmPFC) was found in the presence human } \\
\text { agent advice only: } \\
\text { During the decision phase (run 1, bad advice), GCA } \\
\text { showed that L. aPreC and R. PI were the sources of a } \\
\text { brain network with outputs connections to target ROIs R. } \\
\text { aPreC, PCC, and L. rIPFC. These effective connectivity } \\
\text { path weights were stronger in the human-agent group } \\
\text { compared to the machine-agent group. } \\
\text { Implication: Greater neural resources were engaged } \\
\text { when making decisions in response to bad advice given } \\
\text { by a human than by a machine. The aPreC and PI were } \\
\text { most probably involved in an integration of social } \\
\text { evaluations (judgments about others' intentions and } \\
\text { personal traits) with the recruitment of brain } \\
\text { physiological responses. }\end{array}$ \\
\hline
\end{tabular}


Table IV: Brain regions with putative roles in decision making. [Source: Table 1, Drnec et al. (2016)]

\begin{tabular}{|c|c|c|}
\hline Neural substrate & Role in decision making & Reference \\
\hline Amygdala & Processes/computes the value of negative stimuli & Yacubian et al. (2006) and Basten et al. (2010) \\
\hline Ventral striatum & Processes/computes the value of positive stimuli & $\begin{array}{l}\text { Yacubian et al. (2006), Basten et al. (2010) } \\
\text { and Lim et al. (2011) }\end{array}$ \\
\hline Ventral medial prefrontal cortex (vmPFC) & $\begin{array}{l}\text { Calculates the difference of value signals from } \\
\text { amygdala and ventral striatum in value based decisions }\end{array}$ & Basten et al. (2010) and Philiastides et al. (2011) \\
\hline Dorsolateral prefrontal cortex (dIPFC) & $\begin{array}{l}\text { Calculates the difference of signals from amygdala } \\
\text { and ventral striatum in perceptual decisions }\end{array}$ & Basten et al. (2010) and Philiastides et al. (2011) \\
\hline \multirow[t]{2}{*}{ Lateral intraparietal cortex (LIP) } & $\begin{array}{l}\text { Accumulates and integrates the value of evidence } \\
\text { processed by the vmPFC (evidence largely } \\
\text { from monkeys) }\end{array}$ & $\begin{array}{l}\text { Platt and Glimcher (1999); Platt (2002); Basten } \\
\text { et al. (2010) and Rorie et al. (2010) }\end{array}$ \\
\hline & $\begin{array}{l}\text { A cortical area involved in gaze fixation, saccade, } \\
\text { and attention, underlying evidence accumulation }\end{array}$ & Coe et al. (2002) and Goldberg et al. (2006) \\
\hline
\end{tabular}

A.

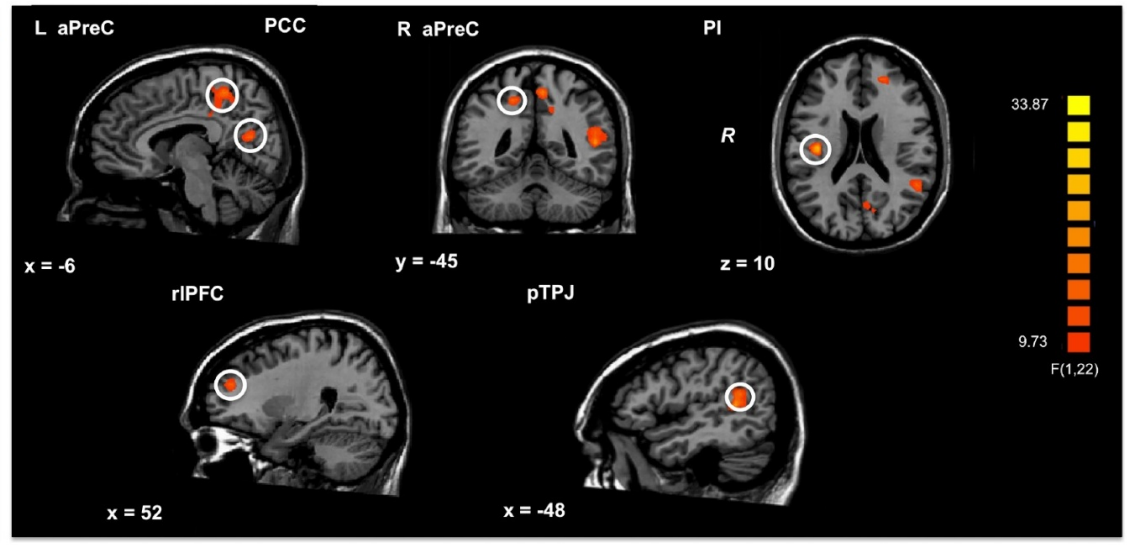

C. LG

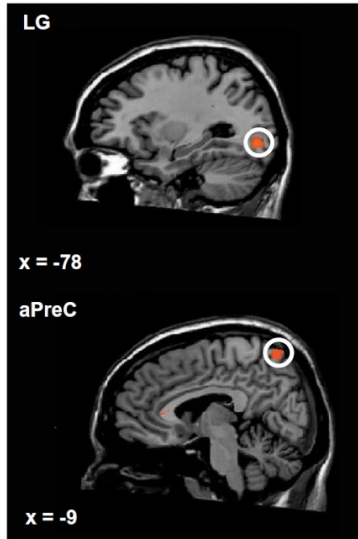

ACC

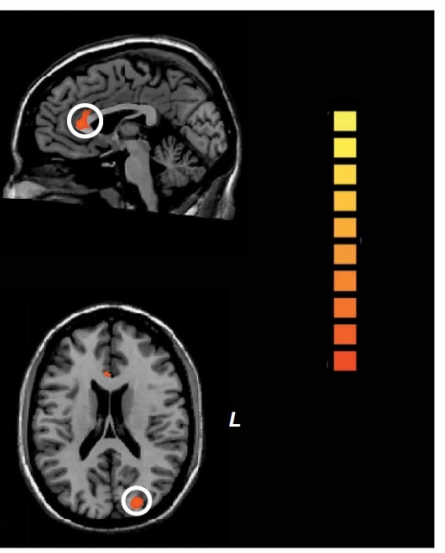

B.

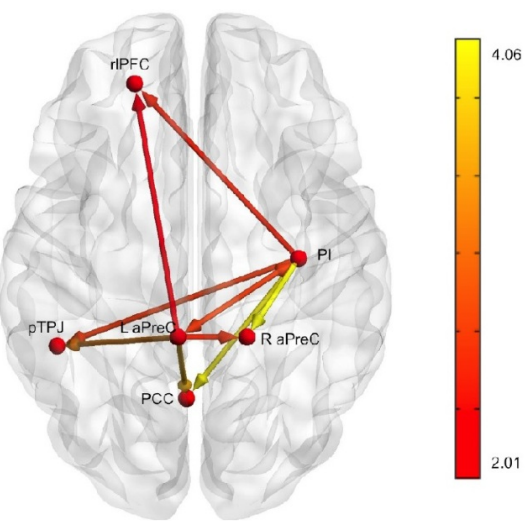

D.

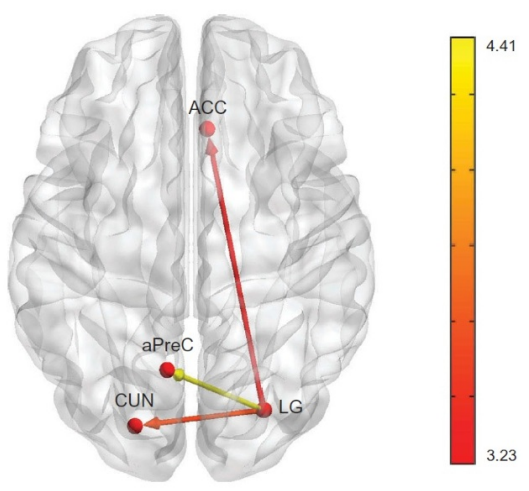

Figure 3: (A) Brain regions activated during the decision phase in a X-ray luggage scanning task with $40 \%$ false alarms based on human-to-machine agent contrast analysis. (B) Effective connectivity network during the decision phase in a X-ray luggage scanning task with $40 \%$ false alarms based on human-to-machine agent contrast analysis. [Source for panels A and B: Figures. 5 and 7, Goodyear et al. (2016) [42]]. (C) Brain regions activated during the decision phase in a X-ray luggage scanning task with $40 \%$ misses based on human-to-machine agent contrast analysis. (D) Effective connectivity network during the decision phase in a X-ray luggage scanning task with $40 \%$ misses based on human-to-machine agent contrast analysis. [Source for panels C and D: Figures. 3 and 5, Goodyear et al. (2017) [43]] 


\section{AI AND EXPLAinAble AI (XAI)}

In contemporary times, AI has achieved unprecedented success in solving increasingly complex real-world problems from many sectors - ranging from facial recognition, natural language processing, and finance to safety-critical self-driving and medical diagnosis [48]. Currently, AI is powering cutting-edge research in the sciences and interface development between AI and the the sciences.

While AI demonstrates tremendous benefits, it cannot explain how they make specific decisions and plan for actions in a comprehensible way to human users (e.g., air traffic controllers, medical doctors). Henceforth, the Defense Advanced Research Projects Agency (DARPA) in the USA created an ambitious XAI Program [49] that commenced in 2017 for exploring the theories for create AI systems whose learned models and decisions can be made comprehensible and trusted by end users. As shown in Figure 4, there has been a yearly growth in the number of AI-focused research works over the past decade, especially in the realm of XAI, which showed marked increases in research outputs from 2018 onwards.

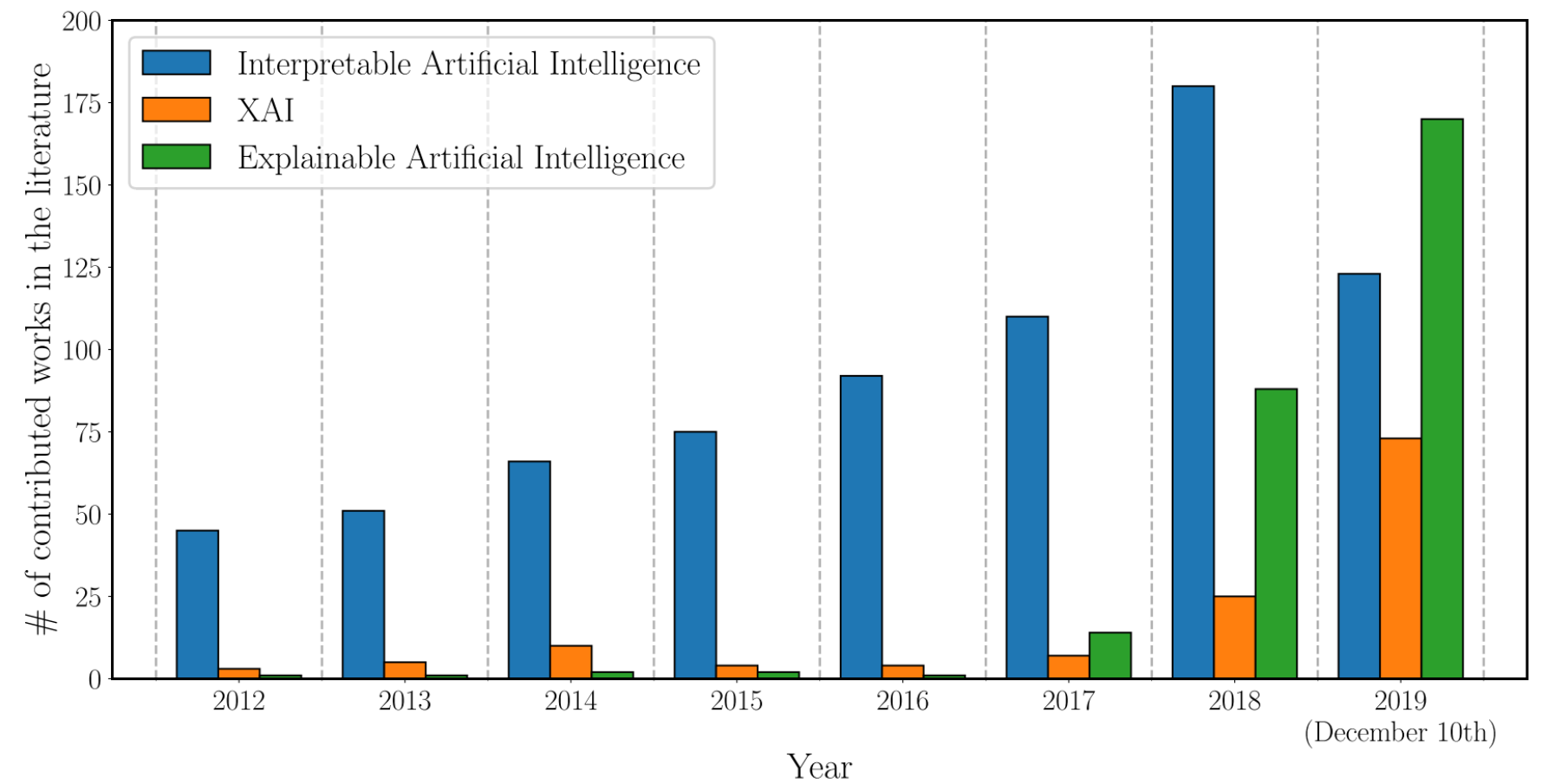

Figure 4: The trend of explainable AI research, progressing from interpretability toward explainability. Source: Figure 1 in [50]. Note that "XAI" and "Explainable Artificial Intelligence" refer to the same thing and are search terms used in the literature search.

\section{A. Opening the Black Box using XAI}

Since the second half of the last century, AI has evolved from a simple rule-based algorithm to a machine being able to learn on its own [51]. In the 1950s, Alan Turing explored the mathematical possibility of Artificial intelligence. He came up with the framework in which he discussed how to build intelligent machines and how to test their intelligence [52]. However, during that time, AI was built upon a rule-based system in which a machine follows a set of rules to come up with the desired output(s) [53]. Due to the fact that a rule-based system does not have learning capability, it cannot adapt to changes when a different input is fed into it. As time passed, computer scientists created algorithms to allow the machine to learn based on the features of the input in order to deliver an appropriate output. By doing so, this covered the weakness entailed by the rigidity of a rule-based system, and rendered learning and adaptability functions to the machine. During the digital revolution that started in the 1980s with the internet, big data has become easily accessible and obtainable, and AI started to develop expertise in specific domains that extend beyond the capability of humans. A prime example is AlphaGO, which was created by Google DeepMind using an artificial neural net architecture. In 2016, AlphaGo defeated 18-time Go (Weiqi) World Champion, Lee Sedol, four games to one [54]. Following on the footsteps of AlphaGo, DeepMind developed AlphaZero, the most powerful Chess engine to date, with the same type of neural net architecture built for AlphaGo combined with two types of advanced algorithms: Monte Carlo tree search and self-play reinforcement learning [55], [56]. By feeding information from two types of neural networks - (i) a policy network that computes the possibility of future moves and (ii) a value network that considers the 
positional outcome of a particular chess variation - into a Monte Carlo tree search algorithm that selects moves and outcome positions with the highest odds of winning, AlphaZero was able to widen its search for optimal chess moves with much greater speed and efficiency compared to extant chess engines like Stockfish that relies on rule-based alpha-beta tree search (pruning) algorithms [55], [56]. The superiority of the AlphaZero's AI architeciture was demonstrated when it beat Stockfish 8 in a 1,000 game match in 2018 with a score of 155 wins, 6 losses, and 839 draws [57].

Even though a rule-based system is rigid, it is transparent and comprehensible as to how its outputs are generated compared with neural networks that power AlphaGo and AlphaZero. While a machine learning (ML)-based architecture or model which a neural network is built upon - has the ability to adapt to task demands and learn rapidly through the fine-tuning of parameters built as part of its functional system, how it made its decisions and generated its outputs can remain opaque even to its own programmers [55]. As such, a conventional ML system can be seen as a "black box" in terms of the interpretability of output generation.

As far as to how AI can mimic humans, it cannot totally replace humans. This is because AI lacks the elements of creativity, ingenuity, and human nature that humans possess. Unless AI start to possess these human traits, humans and AI will have to work hand-in-hand with each other. For human machine operators, it is important to embrace AI as AI can generally provide advice that are much more accurate than a human's, leading to the most appropriate solution to a question [58].

Despite the advantages of using AI, AI has also introduced elements of uncertainty into the decisions it made. As mentioned earlier, an AI model is analogous to a black box that cannot explain how it comes up with a decision. This creates concerns among AI users that they will not be able to make the most accurate decisions due an imperfect understanding of the algorithms. Such a lapse in AI comprehension among users can lead to unfair, biased, or erroneous decision-making [59].

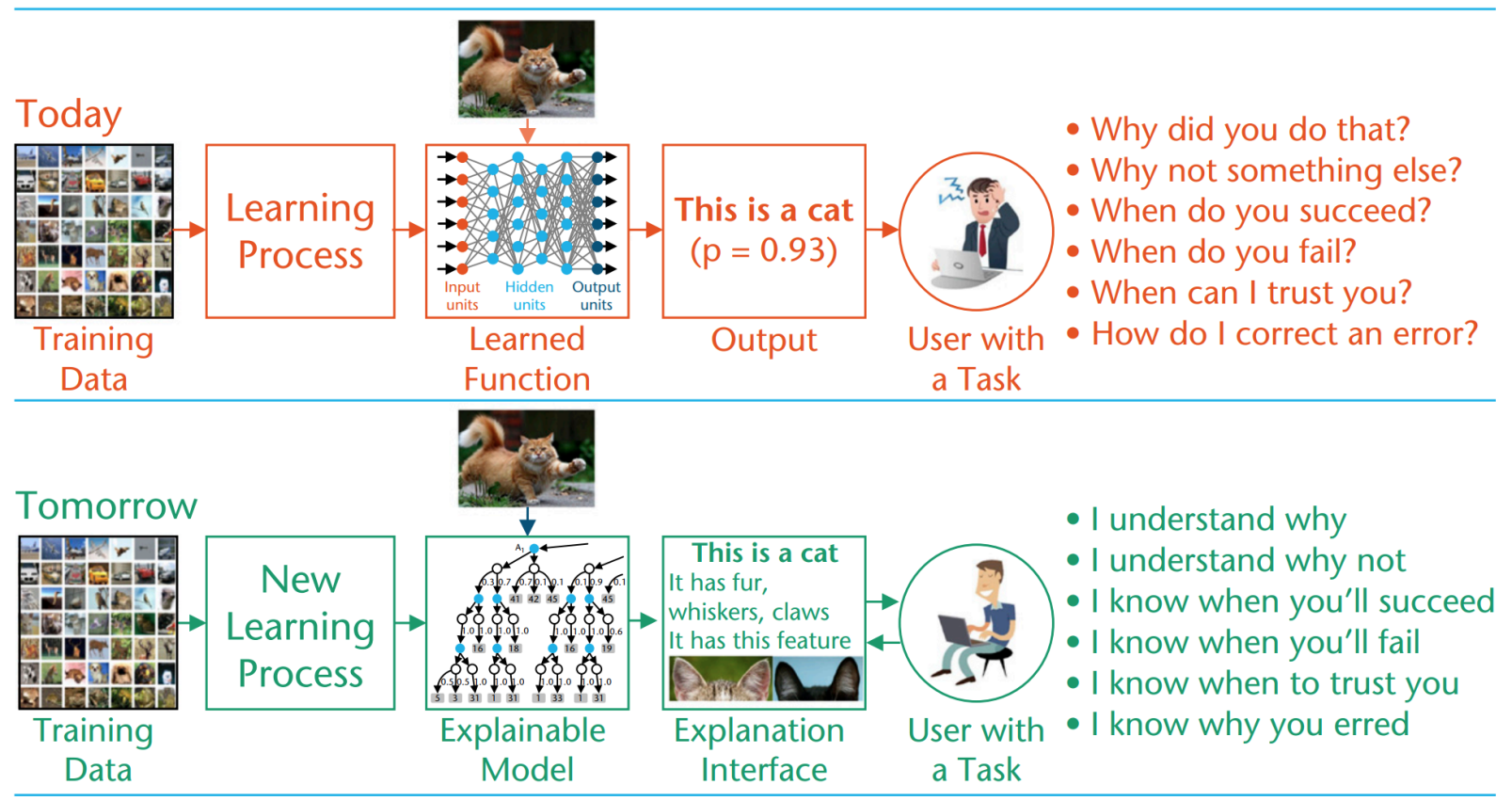

Figure 5: The Explainable AI (XAI) program in DARPA aims to create a suite of machine learning (ML) techniques that: produce more explainable models, while maintaining a high level of learning performance (prediction accuracy); and enable human users to understand, appropriately trust, and effectively manage the emerging generation of artificially intelligent partners. Source: Extracted from [49].

This is where Explainable AI (XAI) comes in. XAI provides the next stage of human-machine collaboration as it explains why AI has reached a specific decision [50]. XAI can help human machine operators to make faster and more accurate decisions and uncover biases in the use of any existing ML system. By doing so, XAI can build "bridges of trust" between humans and machines. Being able to understand how an AI system works is essential to its improvement and practical utilization [50] and Figure 9 shows the promises the XAI of tomorrow can bring, as conceptualized by DARPA [49]. XAI development requires an inclusive design of AI systems, which takes the needs of different end-users into consideration during the AI development. As shown in Fig 6, different stakeholders of AI in ATM (e.g., ATCOs, pilots, passengers, ATMRI, CAAS) will harness XAI over a range of diverse purposes. In Fig 7, Professor Sargur Srihari summarized the reasons for why XAI is needed for system justification and improvement, compliance to legislation, and scientific discovery. 


\section{Who cares?}

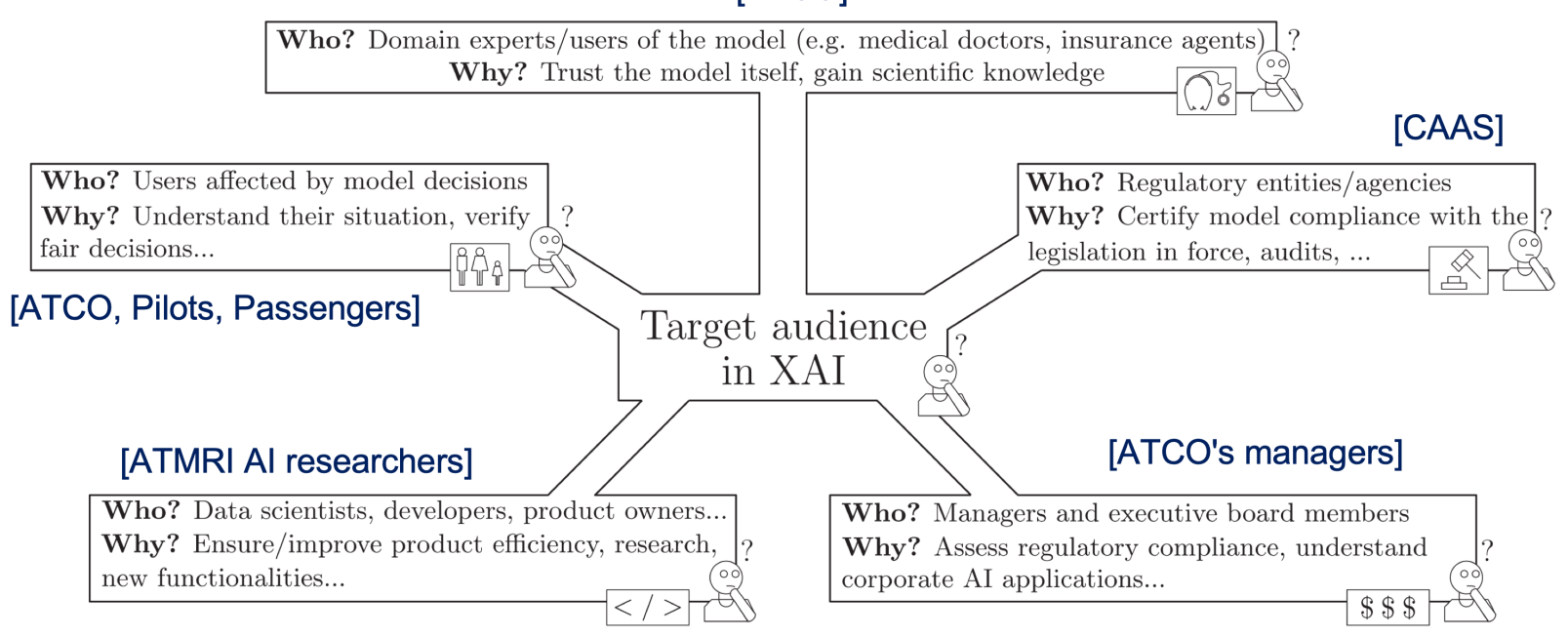

Figure 6: Different audiences and users of AI systems require AI explainability for different purposes (e.g., trust, understanding, legal compliance). We also adopt these concepts for the stakeholders of ATM. Source: Extracted from [60].

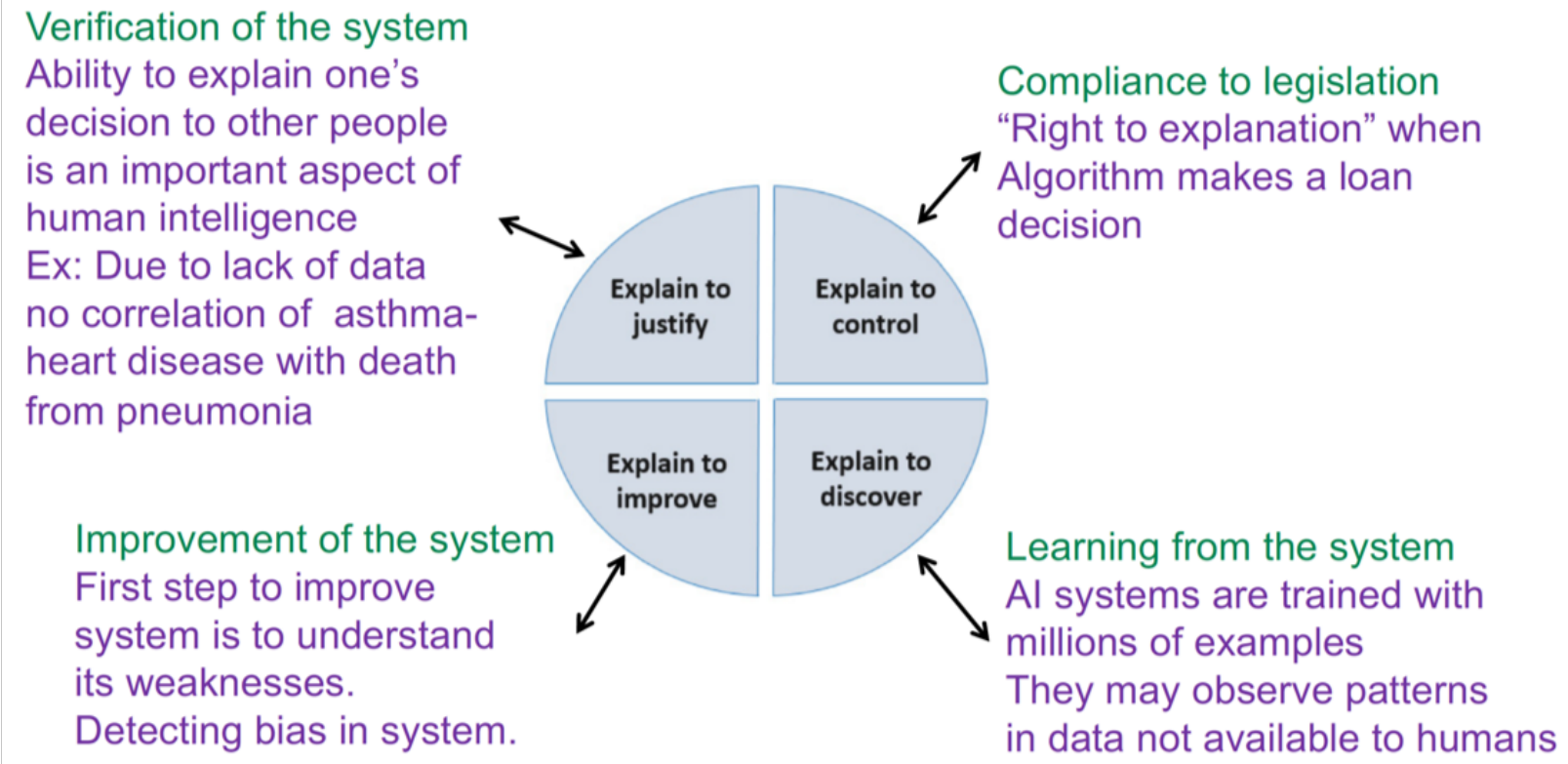

Figure 7: Summary of needs for explainable AI. Explanation is required for justification, improvement, control and discovery of knowledge learned from data. Source: Extracted from lecture slides of Prof. Sargur Srihari.

The main challenges of XAI, depending on the operational relevance of its application, can be broadly categorized into data analytics and autonomy problems, as illustrated in Fig 8. These two problems cover the prediction of events of interests and the decision-making policies of autonomous systems. In ATM, the first problem is related to prediction systems (e.g., runway exit and occupancy time), while the second problem is relevant to recommended systems (e.g., conflict detection and resolution, surface movement optimization). As these two problems concern AI-enabled tools for ATCOs, XAI is important for helping ATCOs to manage their AI "partners" effectively and appropriately.

There are a number of ways to achieve XAI, which can be broadly categorized into ante-hoc, post-hoc and transparent models (see Figure 10, details to be provided in the next section). Building upon the categorization, DARPA also lays out new approaches to create a suite of machine learning techniques that aim at producing explanation while maintaining a high level 


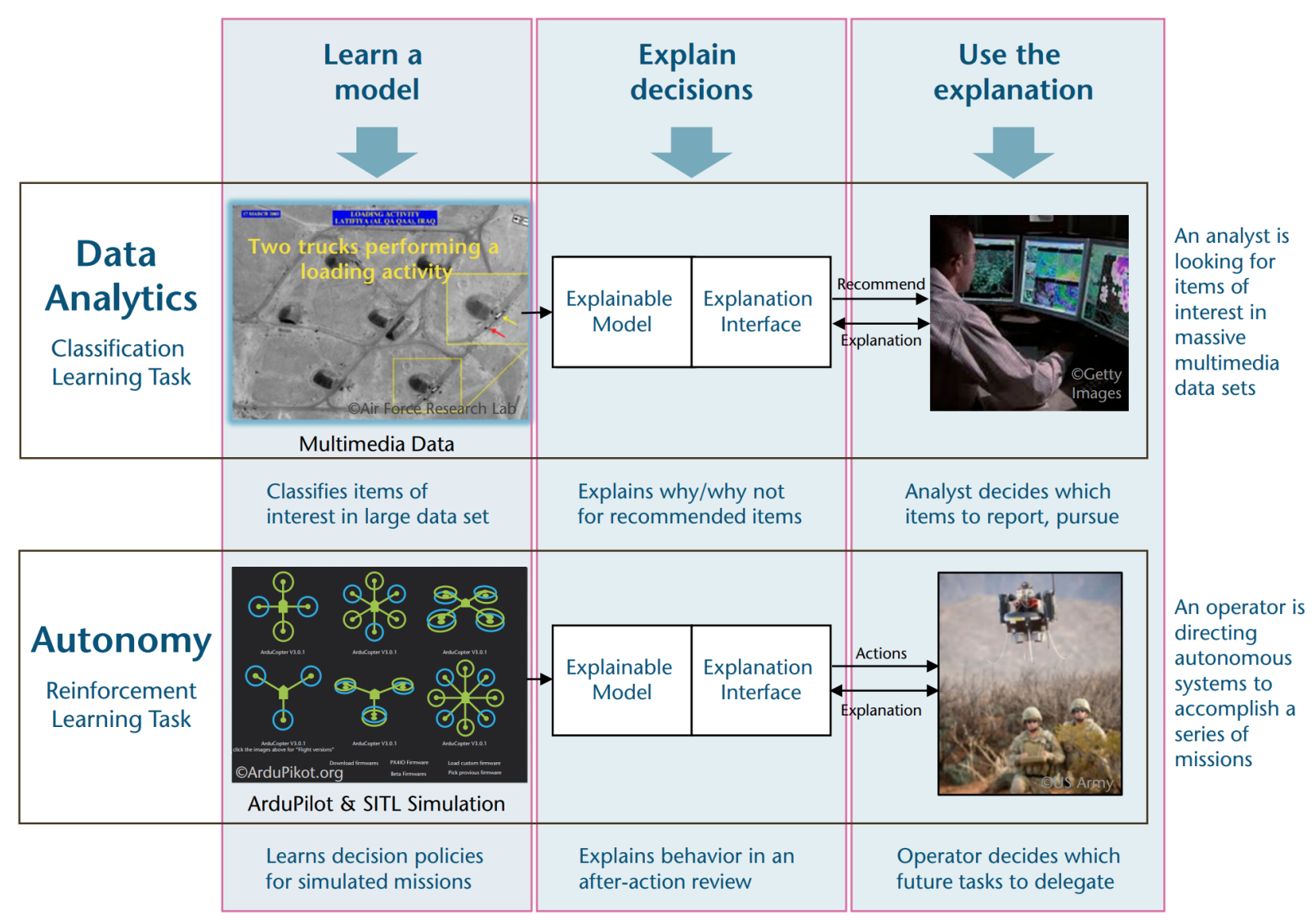

Figure 8: Challenging XAI problem research areas. Source: Extracted from [49].

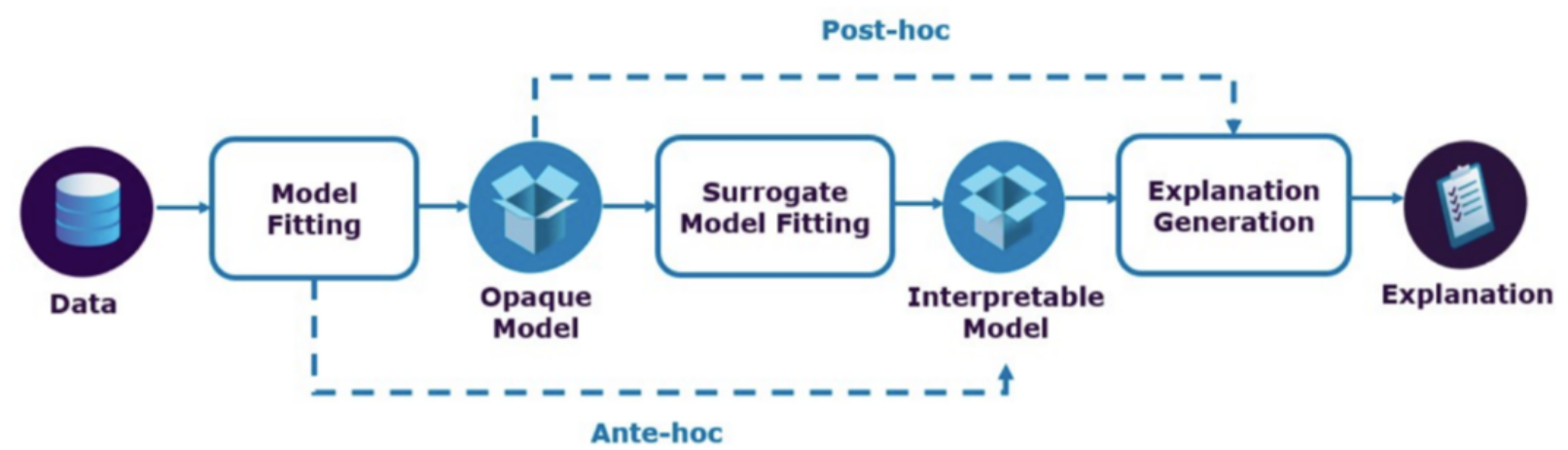

Figure 9: Illustration of different approaches to achieve explainable AI, by developing ante-hoc, post-hoc and transparent models. Source: Extracted from lecture slides of Prof. Sargur Srihari.

of learning performance. Figure 10 shows a notional Venn diagram that clusters machine learning techniques with trade-offs in explainability and learning performance. In this figure, DARPA also outlines three main strategies to achieve XAI, which include deep explanation, interpretable models and model induction.

\section{B. Taxonomy of XAI Techniques}

As shown in Figure 11, there are different ways to get explanations from a ML model. It is either that the model itself is transparent or that there is a need to use post-hoc explainability techniques on an already developed model to generate understandable information with respect to its prediction for any given input. Under post-hoc explainability techniques, there are model-agnostic and model-specific techniques. Model-specific techniques are designed with the purpose for extracting 


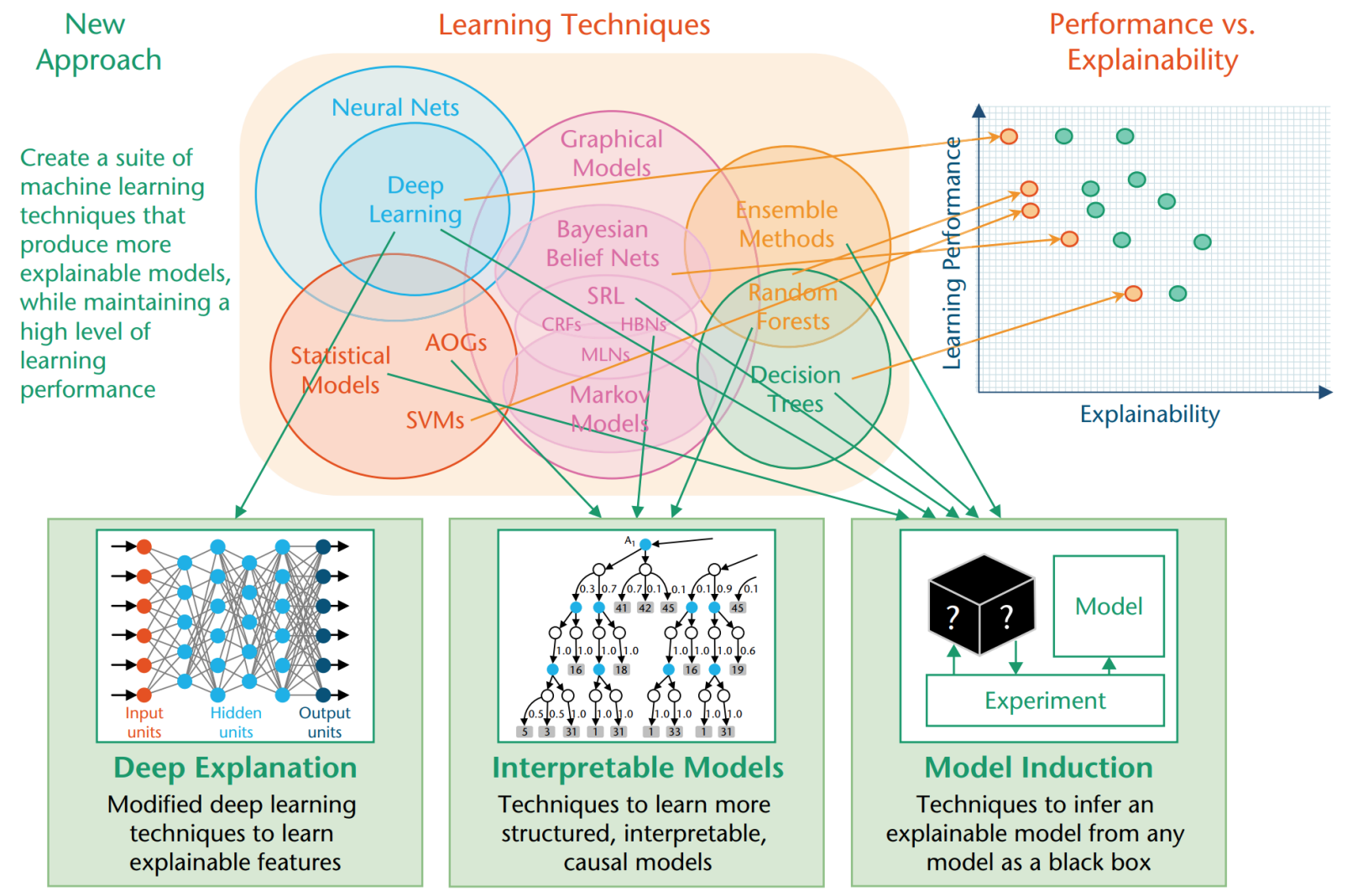

Figure 10: DARPA's strategies to develop XAI. Source: Extracted from [49].

information related to any model from its prediction procedure. By contrast, model-agnostic techniques are dependent on model simplification, feature relevance explanation, local and visual explanation techniques. The subsections below present an overview of the most popular model-agnostic and model-specific techniques. This overview is intended to be brief and comprehensible for a general audience with elementary computer science knowledge. For detailed explanations of these techniques, the reader is advised to refer to the systematic review done by Arrieta et al. (2019) [50].

Starting with model-agnostic techniques, we describe three techniques of interest (in bold) in the following paragraphs. We follow up on these information with a description of some model-specific techniques in the next subsection.

1) Model-agnostic Techniques

Explanation by simplification is based on rule extraction techniques, where simpler explanations of what a model does is provided based on relevant ML rules. One way is to use LIME. LIME is a local interpretable model that focuses on training a local surrogate model to explain individual predictions [61]. Basically, a local surrogate model is an interpretable model that is used to explain individual predictions of "black box" ML learning model [62]. With reference to Figure 11, LIME falls under local explanation techniques. A brief mathematical description of how LIME works is provided in the subsection "Two popular model-agnostic XAI techniques" below.

Feature relevance explanation techniques explain the model by ranking or measuring the significance of each feature in the prediction output. One of the main contributors to this technique is SHAP. The explanation model of SHAP assigns gain and loss values (i.e., "payout" values) to each input feature, in the form of Shapley values, as shown in Figure 14. These values represent and explain three desirable properties of the SHAP model - local accuracy, missingness, consistency [62]. A brief mathematical description of how SHAP works is provided in the subsection "Two popular model-agnostic XAI techniques" below.

Visual explanation techniques use visualization to help explain a black box model. However, visual explanations are not common because they must fit nicely into a ML model without regards for its inner structure. Hence, visual explanation is usually tagged with feature relevance techniques which provide information that is eventually displayed to the end user.

2) Model-specific Techniques

Ensembles and multiple classifier systems include tree ensembles (also known as "random forests"). Tree ensembles offer one of the highest accuracy among ML models. With a single decision tree, the decision flow can be visualized and interpreted 


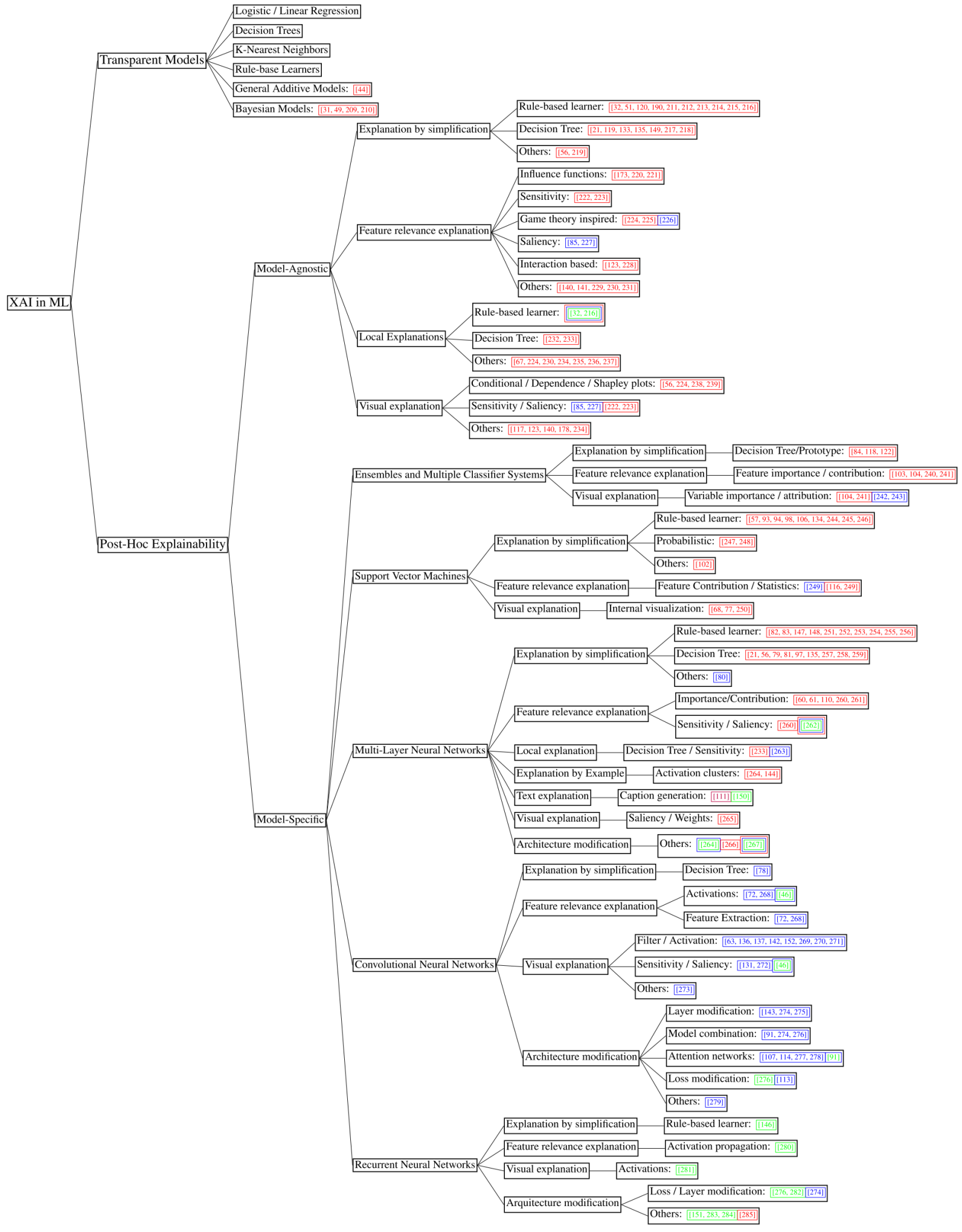

Figure 11: Taxonomy of XAI techniques. Source: Figure 6 in [50]. References boxed in blue, green and red correspond to XAI techniques using image, text, and tabular data, respectively.

easily. However, using it can lead to overfitting. Therefore, tree ensembles combine a multitude of different decision trees to 
overcome the effect of overfitting, but the model can become more complex, which lead to a need for post-hoc explainability techniques. For tree ensembles, they are explained using simplification and feature relevance techniques.

Support vector machines (SVMs) are much more complex than tree ensembles. SVM creates a hyper-plane or set of hyperplanes in high dimensional space and get the one with the largest margin to the nearest training data. Due to the excellent prediction capability of SVM, it is one of the most used ML model. Post-hoc explanations applied to SVMs covers explanations by simplification, local explanation and visualisation, and example. There are several research studies that contributed to the explanation of SVMs. These studies applied rule-based explanations [63], fuzzy rules [64], or added extra hyperplanes to the model [65]. In post-hoc techniques used in SVMs, local explanation technique is used frequently, unlike other models that applied model simplification as their preferred post-hoc techniques. As mentioned in the "Trust-related Human Performance" section above, SVM has been used as the model of choice in the classification of EEG-based spectral features (obtained from ATCO participants) into different categories of workload during real-time air traffic simulations [25], [34].

In the section below, we discuss deep Learning (DL), a subset of ML comprising of multi-layer neural networks, convolutional neural networks and recurrent neural networks.

C. XAI Techniques for Deep Learning

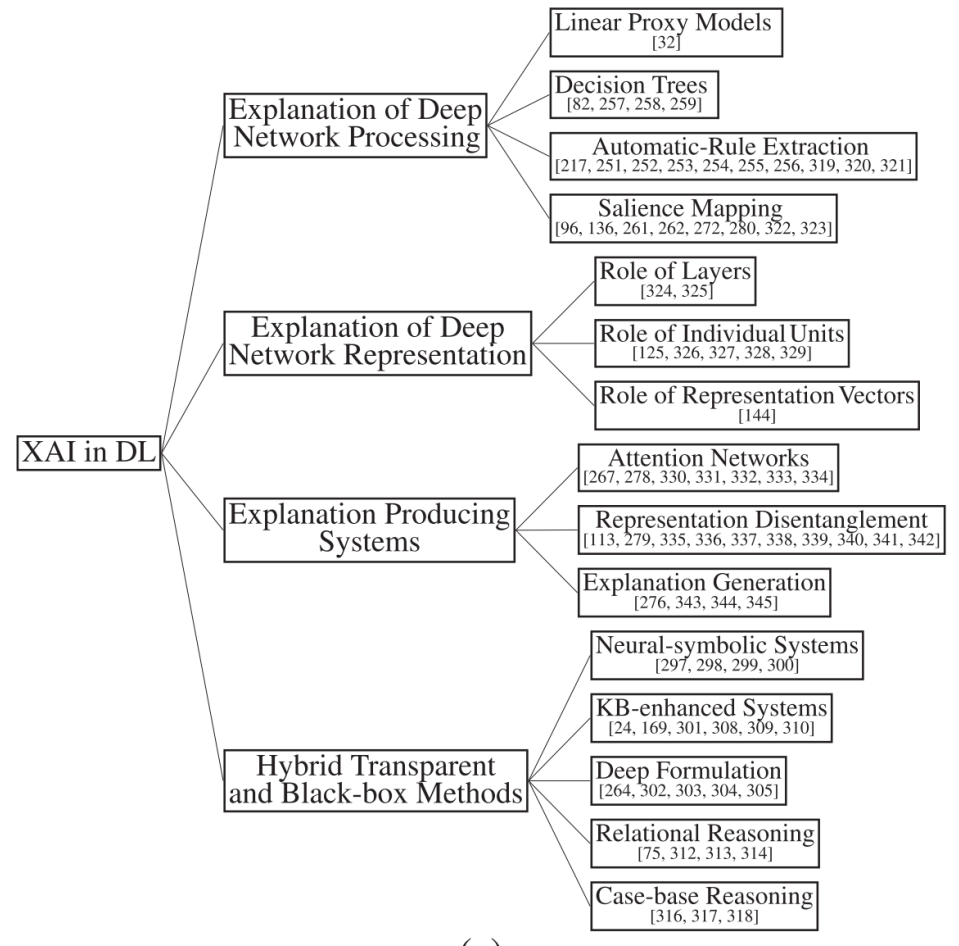

(a)

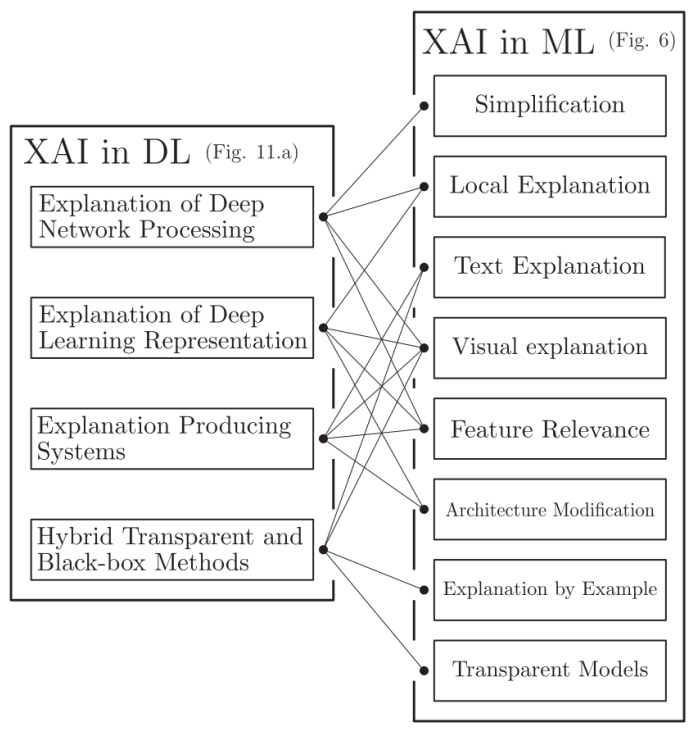

(b)

Figure 12: Taxonomy of deep-learning based XAI and the connection to the general XAI taxonomy. Source: Figure 11, extracted from [50].

Multi-layer Neural Network is a model that contains more than one layer of artificial neurons or nodes and each nodes uses a nonlinear activation function. This allows the model to infer complex relations between variables. Many XAI techniques proposed for multi-layer neural networks tackle a single hidden layer and only a few have tried to focus on multiple hidden layers. Some algorithms in the market include pixel-wise decomposition and Deep Learning Important FeaTures (DeepLIFT) [66]. Pixel-wise decomposition seeks to explain each pixel from an input image as a weighted coefficient in the prediction function $\mathrm{f}(\mathrm{x})$ made by a classifier in an image classification task [67]. DeepLIFT, like pixel-wise decomposition, is a method for decomposing the output prediction of a neural network, but DeepLIFT uses back-propagation on the contributions of all neurons in the network to every feature of the input.

Convolutional Neural Netoworks (CNNs) are state-of-the art models fundamental to computer vision tasks. CNNs are built as a sequence of convolutional layers and pooling layers to self-learn features at higher levels. The outputs created by the model form a feature map that is connected by one or more layers. Due to the map's complex internal structure, it is very difficult to explain how the outputs are formed. As CNNs are mainly used as an extended representation of human cognitive skills, it is easier to understand the visual data as compared to other models. However, this does not mean that XAI is not 
needed for CNNs. CNN decision process via mapping back onto the output and understanding how the intermediate layers work is still a mystery and many researchers have tried to address these issues. LIME is able to solve part of the black box problem for CNN models by perturbing the input to see how the predictions change[61]. LIME can also create contiguous "superpixels" from the input image and the LIME model can be ran together with the CNN model to get output probability values for such superpixels. By using linear modelling on the dataset, LIME generates superpixels with highest positive weights as an explanation of the outputs from the CNN model [50].

Recurrent Neural Networks (RNNs) are generalizations of feedforward neural networks that have internal memories. RNNs perform the same function for every instance of data input while the output of the current input depends on the past computation of a previous input. After an output is produced, it is copied and sent back into the recurrent network. The decision process is dependent on the current input as well as an output that learned from the computation of a previous input. Extant studies have investigated the explainability of RNN models by understanding what a RNN model has learned and by modifying RNN architecture to understand the decision they make [50].

Knowledge Base (KBs) combines knowledge that is diverse but not limited by a specific domain and the level of complexity of knowledge in a specific field. It is a hybrid approach that Uses background knowledge in the form of logical statements or constraints in KBs has shown that it helps to improve explainability and performance with respect to purely data-driven approaches. The KB model has been shown to be able to jointly learn and reason with both symbolic and sub-symbolic representations and inferences [68]. Another approach for a hybrid model like the KB model is to enrich a black box model with information from a "white box" system that is more transparent and interpretable. An example is to combine a neural network with a transparent Case Based Reasoning (CBR) system, a method that has been shown to improve interpretability while keeping the same accuracy in model prediction [69].

\section{Two Popular Model-agnostic XAI Techniques}

1) LIME

Local Interpretable Model-Agnostic Explanations (LIME) is a local interpretable model that are used to explain individual predictions of black box ML models. LIME focuses on each individual prediction and provides explanations that can lead to a better understanding of the feature contribution instead of focusing on general predictions. The explanation produced by LIME is obtained by the following mathematical equation (1):

$$
\operatorname{explanation}(x)=\operatorname{argmin}_{g \in G} L\left(f, g, \pi_{x}\right)+\Omega(g)
$$

The explanation model for instance $\mathrm{x}$ is the model $\mathrm{g}$ that minimizes loss, which measures how near the explanation is to the prediction of the black box model $f$ while the model complexity $\Omega(g)$ is kept to the minimum. G represents the family of possible explanation. The proximity measure $\pi_{x}$ defines how large the neighborhood is around instance $\mathrm{x}$. The idea behind LIME is presented in Figure 13. When other sample instances lie close to $x, x$ will have a high weight due to the contribution of $\pi_{x}$. Alternatively, when those instances are placed far away from $x, x$ will have a smaller weight due to lower contribution from $\pi_{x}$.

LIME will be explained further below under the sections on model-agnostic and model-specific techniques.

2) SHAP

SHapley Additive exPlanations (SHAP), by Lundberg and Lee (2016) [70], is based on the game theoretically optimal Shapley values, created initially by Shapley et al. (1988) [71]. It is a method to explain individual predictions. SHAP computes Shapley values from cooperative game theory. The feature value of the instance or a group of feature values acts as a "player" in a game and the prediction is the "payout". Shapley values tell us how to distribute the payout fairly among the players. The explanation produced by SHAP can be represented by the following mathematical equation (2):

$$
\operatorname{explanation}\left(z^{\prime}\right)=\phi_{0}+\sum_{j=1}^{M} \phi_{j} z^{\prime}
$$

where:

$z^{\prime} \in\{0,1\}^{M}$ is the coalition vector, $M$ is the maximum coalition size, and $\phi_{j} \in R$ is the feature attribution for feature $j$, the Shapley value.

In the coalition vector, an input value of "1" means that the corresponding feature is "present" and 0 means thhat it is "absent." The Shapley values represent and explain three desirable properties of the SHAP model - local accuracy, missingness, consistency [62]. These properties can be obtained by manipulating the variables in equation (2). 


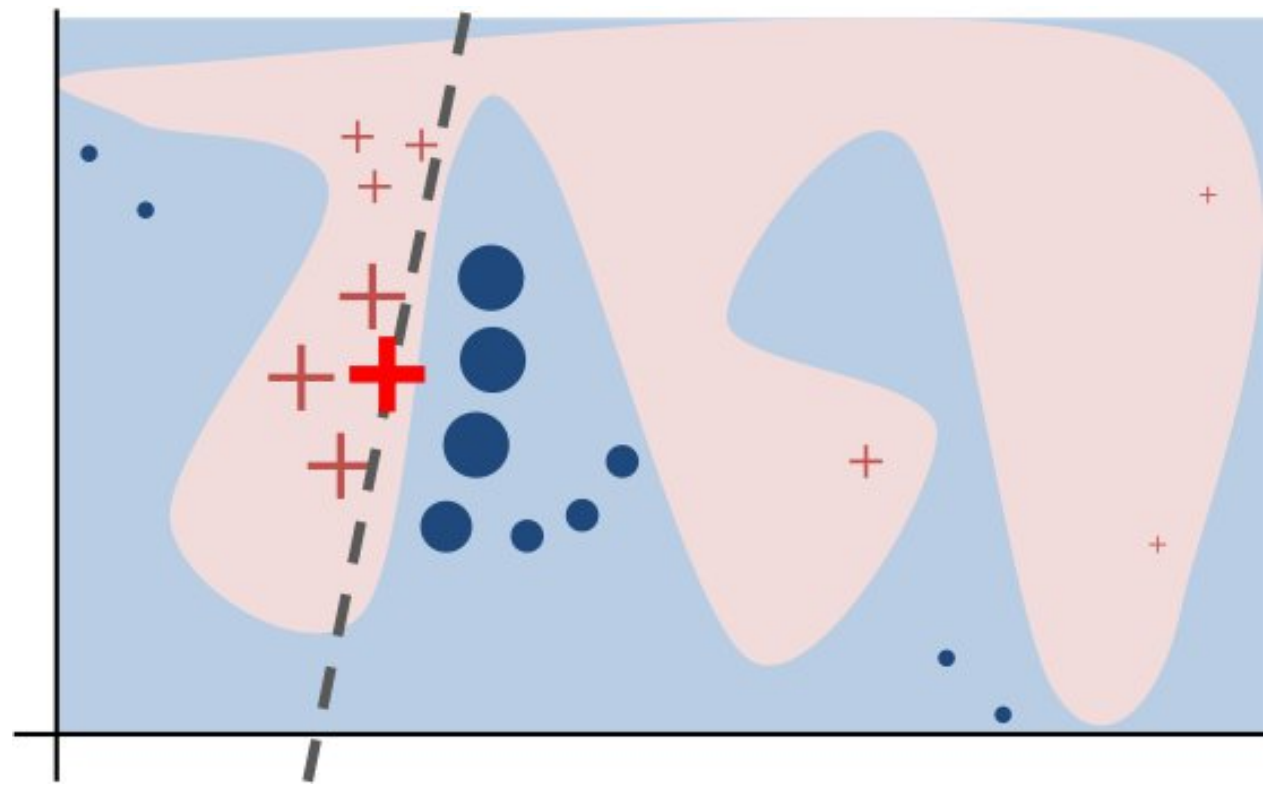

Figure 13: The intuition for LIME. The blue / pink backgrounds represent the complex decision function $f$, which cannot be approximated accurately by a linear model. The bold red cross is the instance being explained. The instances are the output produced from $f$ and the weights to the instances are the proximity to the instance being explained. The dashed line is the local explanation. Source: Extracted from [62].

Local accuracy can be obtained by defining $\phi_{0}=\mathbf{E}_{X}(\hat{f}(z))$ and by setting all $z_{j}^{\prime}$ to "1." This will output the Shapley efficiency property.

$$
f(z)=\phi_{0}+\sum_{j=1}^{M} \phi_{j} z^{\prime}=\mathbf{E}_{X}(\hat{f}(Z))+\sum_{j=1}^{M} \phi_{j}
$$

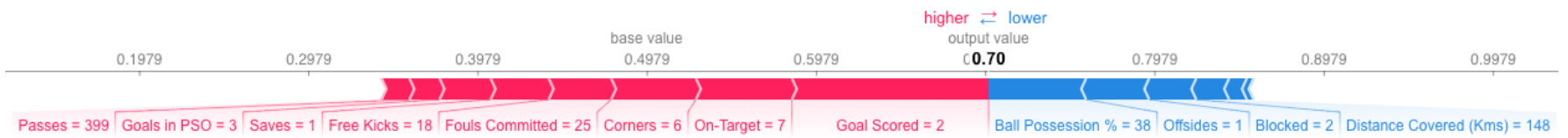

Figure 14: SHAP values to explain the prediction of a soccer player getting number of goals. The base value of 0.4979 and the predicted value of 0.70 . The pink bar indicates which feature values increases the predictions and their visual size shows the magnitude of the feature effect. The blue bar indicates which feature values decreases the predictions. The feature that impact the prediction the most comes from the goal scored being "2." This illustration is taken from an online website.

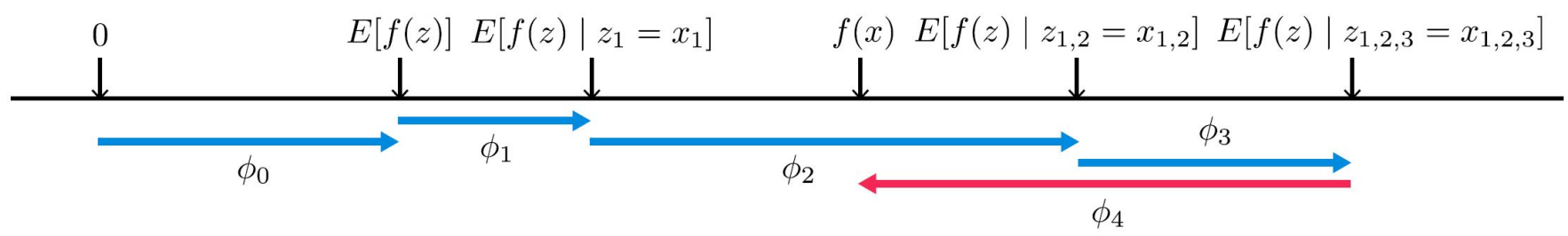

Figure 15: Illustration of the feature contributions leading to the final output, $f(x)$. Source: Extracted from [72].

Missingness is the missing feature gets an attribution of zero. When $z^{\prime}=1$, it shows that the feature value is present while "0" means that the feature value is missing from the instance of interest. According to Lundberg's theory in a "minor book- 
keeping property" [70], a missing feature can have an arbitrary Shapley value without affecting the local accuracy property since it is multiplied with $z^{\prime}=0$.

$$
z^{\prime}=0 \Rightarrow \phi_{j}=0
$$

Consistency states that if a model changes with the marginal contribution of a feature value increasing or staying the same, the Shapley value should correspondingly increase or stay the same. This can be explained via equations (5) and (6).

Let $f_{x}\left(z^{\prime}\right)=f\left(h_{x}\left(z^{\prime}\right)\right)$ and $z_{j}^{\prime}$ indicate that $z_{j}^{\prime}=0$. For any two models $f$ and $f^{\prime}$ that satisfy:

$$
f_{x}^{\prime}\left(z^{\prime}\right)-f_{x}^{\prime}\left(z_{j}^{\prime}\right) \geq f_{x}\left(z^{\prime}\right)-f_{x}\left(z_{j}^{\prime}\right)
$$

and for all inputs $z^{\prime} \in\{0,1\}^{M}$, then:

$$
\phi_{j}\left(f^{\prime}, x\right) \geq \phi_{j}(f, x)
$$

\section{Human-Ai TEAming}

In the current implementation of AI in the ATM domain, human intervention is always needed because existing AI systems built for ATM have only reached the stage of supporting human operators in data acquisition and anaysis (Levels 2 and 3 in Figure 16). The reasoning and decision-making skills of ATM-directed AI have not been well-researched. Without a welldeveloped AT that can reason and explain its "thought" processes, human decision-making will always be required for any ATM-related task. Without explainability in the AI, human operator will not be able to fully comprehend how the AI model generated the predicted outputs and consequently, any trust established between the human operator and the AI will not be strong. With the integration of XAI into ATM operations, human operators will be able to perceive the AI's "train of thoughts" - and this can facilitate the building of human-AI trust.

\section{A. Levels of Automation (LoA)}

\section{B. Human-in-the-loop XAI}

The past recent years have witnessed a noticeable growth in the volume of research in the XAI domain (Figure 4). In a recent XAI experiment conducted by Yang et al. (2020) [74], participants classified the category that a leaf belongs to using a XAI classifier that provided explanations and a non-XAI classifier that did not provide such explanations. Yang et al.'s (2020) findings showed that the visual explanation technique improved user trust in machine learning. The results also showed that the use of grid explanation improved user trust and classification performance to a larger extent than the use of tree and graph explanation.

In another study conducted by Lundberg et al. (2017) [75], the surgeon used the explanations provided by XAI during a real-time operation to prevent the patient from going into hypoxemia. Hypoxemia is a situation in which the patient has low arterial blood oxygen level and it can be fatal to the patient during surgery. During the surgery, real-time physiological information from the patient was inputted into the XAI model. The model predicted hypoxemia events and explained why such an event might occur by displaying how much each feature affected the outcome of the undesired event.

Here, it must be emphasized that there is an increasing demand for XAI systems in the medical domain because in order for any AI system to maximally support the decision-making process of the human medical professional, it must have the capacity to re-enact the machine decision-making process and/or to reproduce the knowledge extraction process [76]. Over the past recent years, a XAI technique that has sparked interest in the medical subdomain of digital pathology is the use of deconvolutional networks that enables the unsupervised construction of hierarchical image representations and visualization of convolutional network layers [77]. For instance, through the use of Amplitude Modulation-Frequency Modulation (AM-FM) decompositions, meaningful reconstructions of medical images and video contents can be attained. This technique works by decomposing input medical images into a sum of bi-dimensional AM-FM harmonic signals that can be used to provide graded visualizations of dangerous components (e.g., plaques) on a biological surface (e.g., inner surface of an artery) over different degrees of FM reconstructions [76]. This form of multi-scale AM-FM decompositions have been applied to the screening of diabetic retinopathy and the assessment of images collected from hyteroscopy, fMRI, and atherosclerotic plaque analysis [76]. 


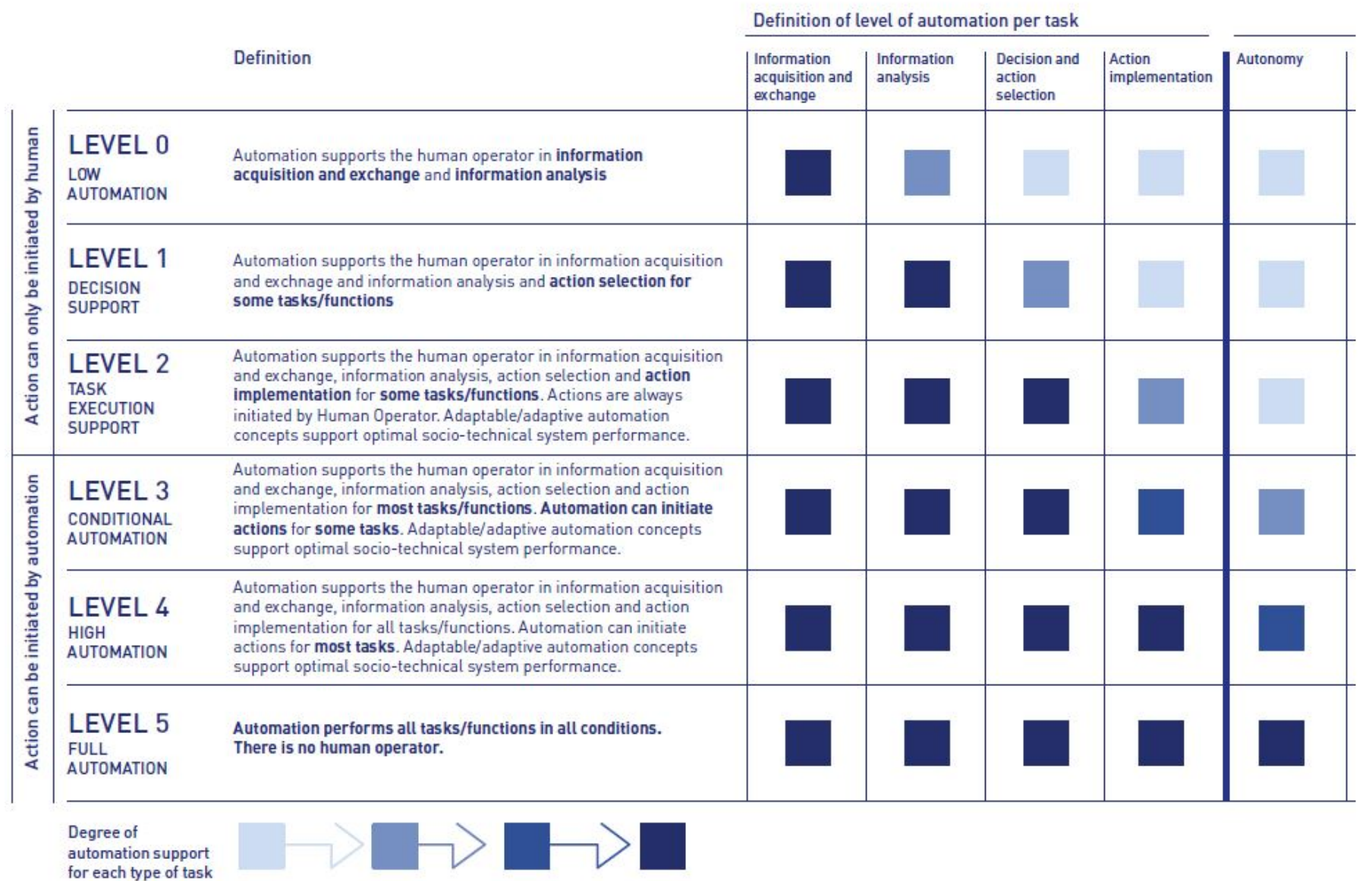

Figure 16: Illustration of a Level of Automation (LoA) model oriented toward ATM and ATCOs used currently by SESAR [73]. This model contains six levels, progressing from Level 0 , low automation, to Level 5, full automation.

\section{Discussion}

\section{A. Neuroscience-inspired AI}
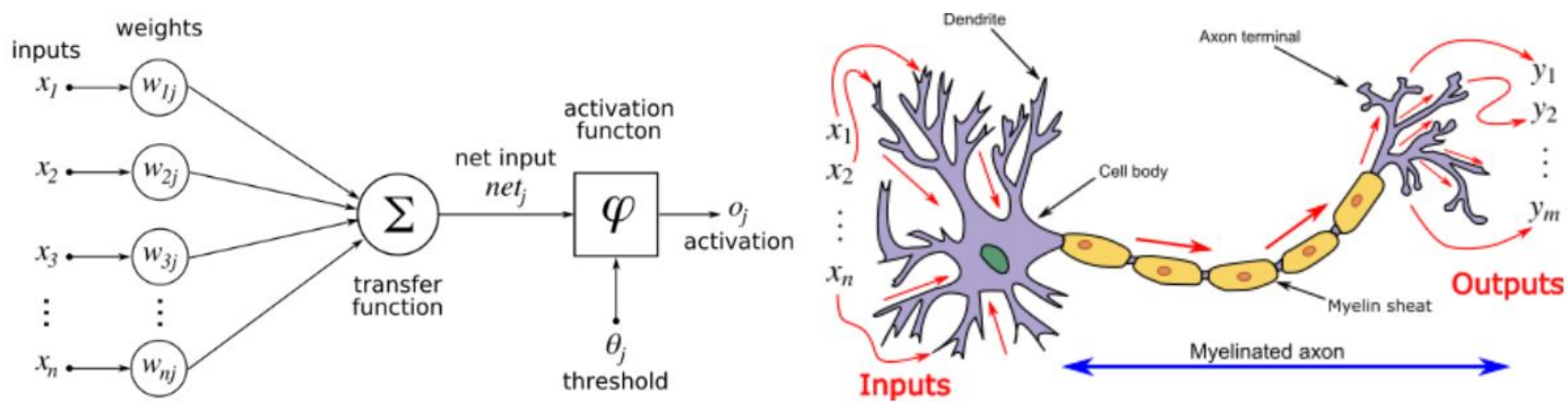

Figure 24: Illustration of a mathematical function being conceived as a model of biological neurons. Source: Wikipedia [81].

Advances in neuroscience research facilitate the design and validation of AI techniques and systems [78]. First, neuroscience inspires the creation of new types of algorithms and architectures complementary to the mathematical methods that have dominated traditional approaches to AI [78]. Second, neuroscience can provide validation of existing AI techniques. If a known algorithm is subsequently found to be implemented in the brain (Figure 24), then there will be support for the implmentation of this algorithm as part of a general framework or theory of human intelligence [78]. Current neuroimaging techniques have 
Measure of Explanation

\section{Effectiveness}

\section{Explanation Framework}

\begin{tabular}{|c|c|c|c|}
\hline & Recc & $\begin{array}{l}\text { Commendation, } \\
\text { Decision or } \\
\text { Action }\end{array}$ & \\
\hline $\begin{array}{c}\text { Explainable } \\
\text { Model }\end{array}$ & $\begin{array}{l}\text { Explanation } \\
\text { Interface }\end{array}$ & & \\
\hline XAI System & & Explanation & decision \\
\hline $\begin{array}{l}\text { The system ta } \\
\text { input from th } \\
\text { task and make } \\
\text { recommendat } \\
\text { decision, or a }\end{array}$ & $\begin{array}{l}\text { kes } \\
\text { e current } \\
\text { es a } \\
\text { tion, } \\
\text { ction }\end{array}$ & $\begin{array}{l}\text { The system provides } \\
\text { an explanation to the } \\
\text { user that justifies its } \\
\text { recommendation, } \\
\text { decision, or action }\end{array}$ & $\begin{array}{l}\text { based on the } \\
\text { explanation }\end{array}$ \\
\hline
\end{tabular}

User Satisfaction

- Clarity of the explanation (user rating)

- Utility of the explanation (user rating)

Mental Model

- Understanding individual decisions

- Understanding the overall model

- Strength/weakness assessment

- 'What will it do' prediction

- 'How do I intervene' prediction

Task Performance

- Does the explanation improve the user's decision, task performance?

- Artificial decision tasks introduced to diagnose the user's understanding

\section{Trust Assessment}

- Appropriate future use and trust

Correctability (Extra Credit)

- Identifying errors

- Correcting errors

- Continuous training

Figure 17: XAI Framework and Measure of Explanation Effectiveness. Source: Extracted from [49].

offered a detailed characterization of the computations occurring in neural circuits that improved our understanding of brain mechanisms. However, current AI systems lacks the capabilities of connecting core mental abilities and concepts with the physical world, which is needed for a human-like AI to construct mental models that guide inferential and prediction processes in the real world [78]. That is why neuroscience research is needed to help AI to achieve a performance that is close to human intelligence.

The extant AI literature shows that recent developments in AI algorithms, models, and architectures have largely been inspired by neuroscience research on human attention, memory, and continual learning (Figures $19-21$ ), as well as by current theories and conceptualizations about how human intelligence and memory systems work (Figures 20 and 21). For examples, recurrent attention models that take "snapshots" of the input image at each step for the purpose of updating of internal state representations were built upon the knowledge that human attention is limited in capacity and needs to switch strategically among different locations and objects [78] (Figure 19A). Deep Q-networks (DQNs) that combines deep learning (DL) with reinforcement learning (RL) were supported by the theorization of complementary learning systems (CLS) that purportedly lies within the hippocampus and neocortex [78], [79]. According to the CLS theory, the hippocampus serves as the encoder of novel information after a single exposure (i.e., one-shot learning) and the neocortex acts as the consolidator of this information during sleep or resting periods. The memory consolidation process is accompanied by reactivation or "replay" of the memory traces or representations in the hippocampus and exchanging such information with the neocortex in a bidirectional feedback loop [79] (Figure 21). This replay and informational exchange represent an reinstantiation of the neural events that accompanied the original learning event. Building upon this replay mechanism, a DQN contains a replay buffer that simulated the transient storage and replay functions of the hippocampus. Parameters tied to this replay buffer can be gradualy adjusted and optimized to capture events that engender high levels of reinforcements or rewards [78]. More recently, the development 


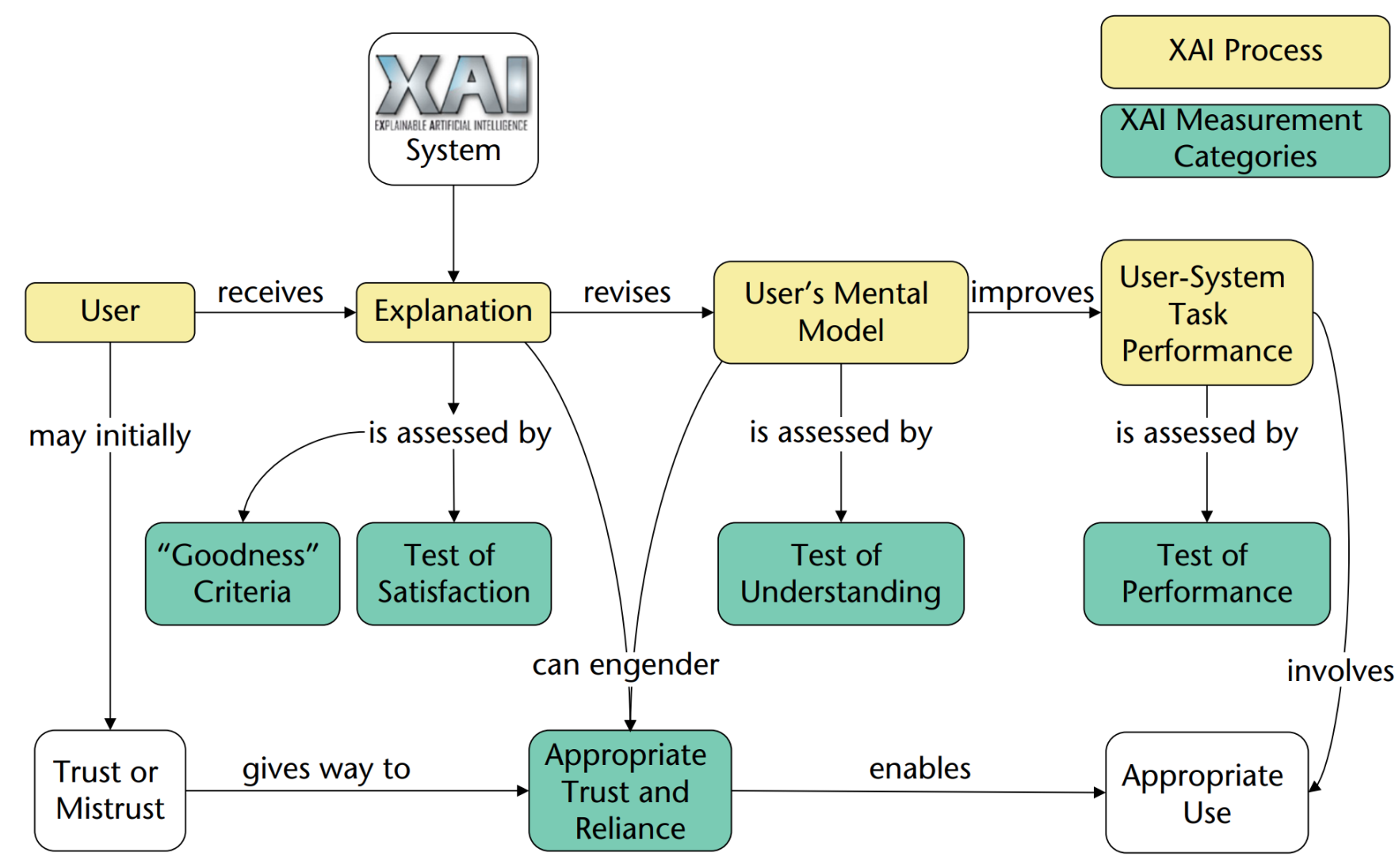

Figure 18: Source: Role of Trust in XAI Process and Measurement. Extracted from [49].

of deep generative models, whose functions mimicked those of the hippocampus in binding together multiple stimuli to create an imagined experience that is both spatially and temporally coherent, has led an AI to possess the human-like ability in making inferences or generalizations from an initial exposure to a small number of examples or concepts (Figure 20D) and in generating new scenes that preserve the geometric features of a newly experienced environment (Figure 20E). In addition, extant knowledge of the biological functions of grid cells in the entorhinal cortices of both rodents (Figure 22) and humans (Figure 23) has led to the development of a DL recurrent network that enabled agents to perform vector-based navigation (i.e., path integration) and shortcut-finding in unfamiliar and changeable spatial environments [80].

\section{B. AI Techniques for Neuroscience Research}

The examples above showed how an improved neuroscientific understanding of how the mind and brain work has contributed to the AI development. It is also possible to approach this developmental trajectory from the opposite direction and ask how neuroscience-inspired AI techniques, models, and systems can contribute to advancing neuroscience research. With ATM-related research in mind, we witnessed the use of machine learning (ML) algorithms and models such as linear discriminant analysis (LDA) and support vector machine (SVM) as reliable classifiers of ATCOs' EEG-based spectral features (obtained from ATCO participants) into different categories of workload during real-time air traffic simulations [32], [33], [29], [34]. As mentioned in the "EEG Studies" section above, these EEG-based workload indices provide objective assessment of task engagement and perceived workload and are useful for triggering adaptive automation (AA) solutions [33], [25]. This form of ML approach has yet to be performed using BOLD signals or parameter estimates derived from fMRI and it remains to be seen as to how successful model-specific ML techniques like SVM can be in identifying and explaining the mental states of ATCOs through the use of fMRI BOLD parameters as inputs. Such inputs can be obtained from either task-based brain activations (i.e., when an ATCO performs an task related to real-world operations in the fMRI scanner) or resting-state brain activations (i.e., when an ATCO undergo an fMRI scan in a relaxed state and without performing any task of interest).

Keeping in mind the feasibility of using such BOLD parameter inputs for developing ML-based classifiers of ATCOs' mental states or conditions, ML and other relevant AI techniques can also be harnessed to compute reliable indices of ATCOs' mental engagement - related to workload, trust, attention, etc. - using data collected from EEG, fMRI, and any 
A
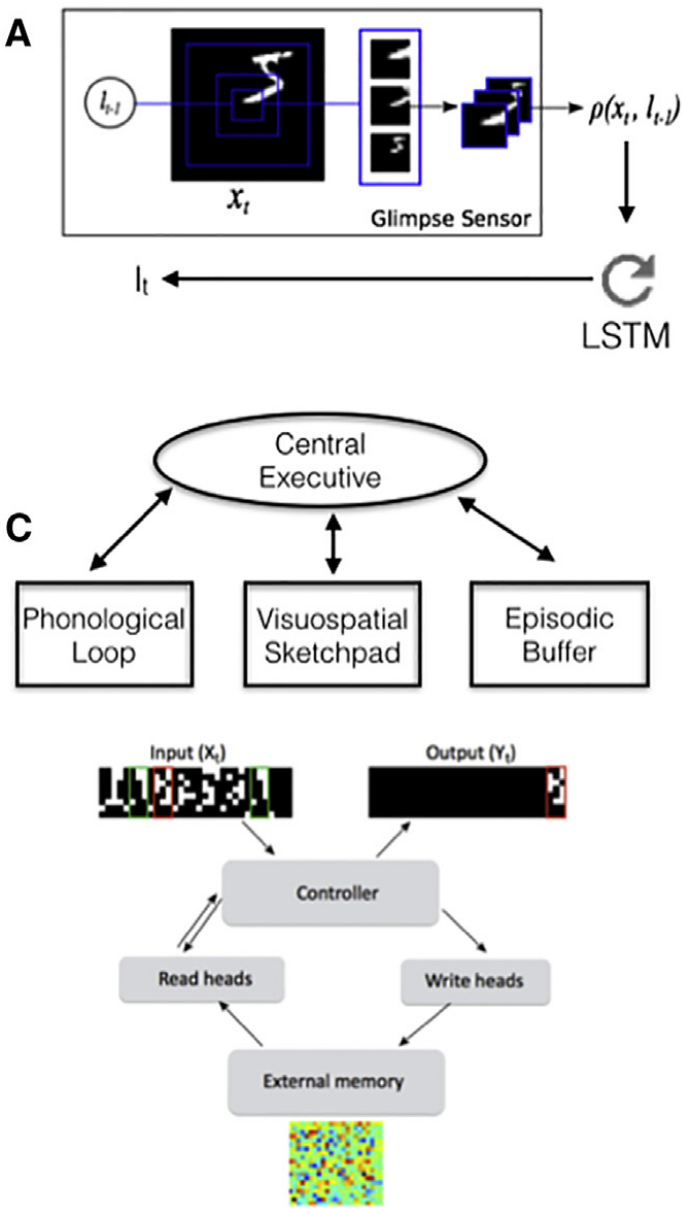

B

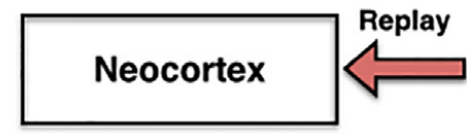

*Slow acquisition of structure

*Parametric

*Efficient representations for generalisation
Hippocampus

*Rapid storage: individual experiences *Non-parametric instance-based system

*Sparse non-overlapping representations (poor generalization)

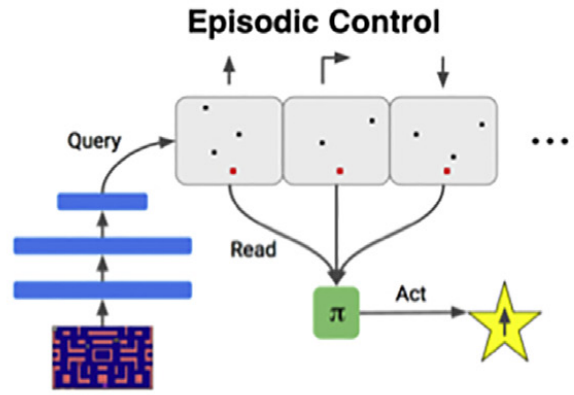

D

Cascade Model

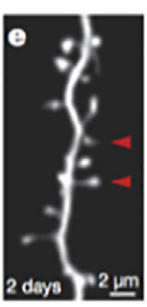

EWC

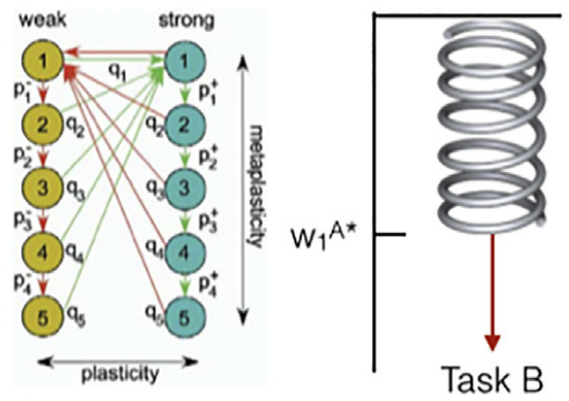

Figure 19: Parallels between AI Systems and Neural Models of Behavior. (A) Attention: Schematic of recurrent attention model. (B) Memory and Learning: Schematic of complementary learning systems and episodic control. (C) Illustration of parallels between macroscopic organization of models of working memory and the differentiable neural computer (Graves et al., 2016). (D) Illustration of parallels between neurobiological models of synaptic consolidation and the elastic weight consolidation (EWC) algorithm. Source: Figure 1 in [78]

other psychophysiological measures. This approach would require the AI algorithm to compute, re-organize, and combine the relevant psychophysiological measures into composite measures that offer higher sensitivity for detecting individual differences in cognition and behavioral performances. Ideally, these composite measures should be used to provide highly objective indicators of ATCO mental states and decision-making behaviors.

In addition to these knowledge about how AI and neuroscience research could faciliate each other's development in the ATM domain, it is worth noting that AI techniques and models are increasingly employed by consumers and providers of healthcare [82], [83], [84]. For instance, SVMs and deep learning neural networks are currently used for the precise classification of patients with brain diseases and in the medical field of radiomics, which requires the detection of clinically relevant features in imaging data beyond what can be perceived by the human eye [83], [84]. Different varieties of AI algorithms, supported by big data, are also being developed and increasingly used to diagnose and recommend medical treatments under situations where radiological image analysis is involved [83].

Furthermore, from a neuroscience researcher's perspective, a promise that AI / XAI offers relates to the creation of automated yet human-like neuroimaging data analysis pipelines that can greatly reduce the time and resources spent on a manual programming of data analysis functions. Owing to the multi-layered complexities of neuroimgaging data analysis, any AI tool developed for automated data analysis should preferably be interpretable with respect to its programmed functions and possess a human-like ability to detect and resolve errors during any processing stage. The development of such powerful AI tools for neuroimaging analysis would not just simplify neuroimaging data analysis and accelerate neuroscience research output, but also inspire the creation of similar data analysis tools in other research domains involving multivariate and big data 

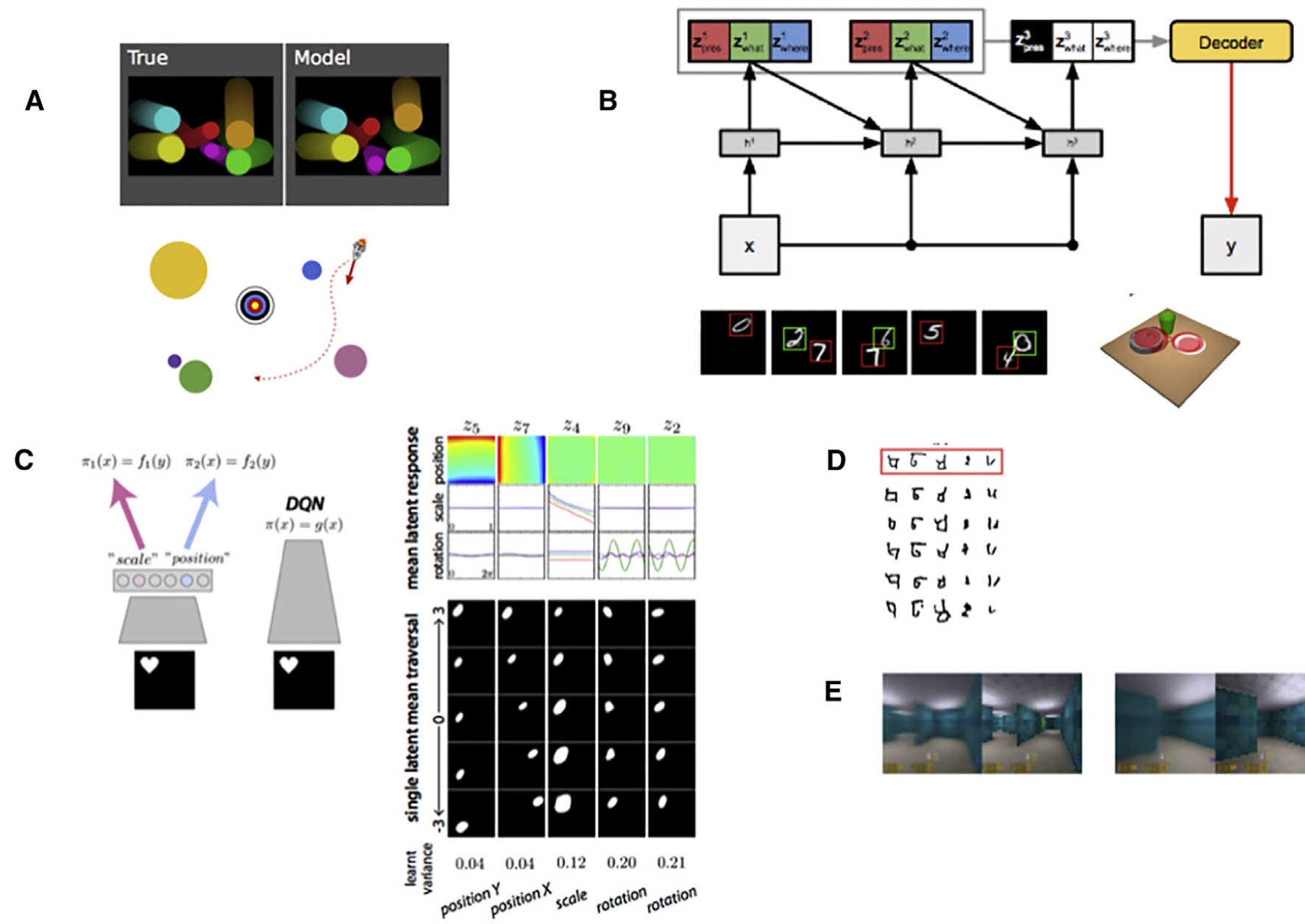

E
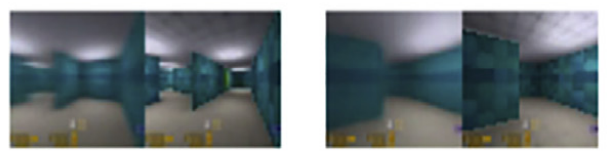

Figure 20: Examples of Recent AI Systems that Have Been Inspired by Neuroscience: (A) Intuitive physics knowledge. (B) Scene understanding through structured generative models (Eslami et al., 2016). (C) Unsupervised learning of core object properties (Higgins et al., 2016). (D) One-shot generalization in deep sequential generative models (Rezende et al., 2016b). (E) Imagination of realistic environments in deep networks (Chiappa et al., 2017). Source: Figure 2 in [78]

analysis.

\section{Conclusion}

In summary, this paper reviewed the most recent neuroscience research studies in the HMI and ATM domains, explained how AI and XAI works, and discussed the relationship between AI and neuroscience in terms of mutually beneficial roles. The overall picture presented was one of upward advancement in the twin engines of AI and neuroscience, each of which had made significant progress over the past few decades. In order for any AI-inspired neuroscience research or neuroscience-inspired AI research to take root, it is vital that researchers and developers working in either discipline find ways to share their ideas and forge collaborations with one another [78]. Communication and collaboration, like trust, are essential "human-in-the-loop" components that shall facilitate the development of new technologies for delivering neuroscience-inspired and trusted AI tools for safe and efficient air traffic services. Crucially, this literature review showed that while a substantial variety of AI and XAI techniques have been developed in recent times, they have not been incorporated widely into ATM-related research, in particular research on human-AI trust. Therefore, much more work concerning human-AI cooperation, teaming, and trust needs to be done in the future and we hope that the information contained herein will provide a good foundation for future research studies in ATM - as well as in other technological domains - that aim to integrate state-of-the-art methods in AI, neuroscience, and human factors.

\section{ACKNOWLEDGMENT}

This research is supported by the National Research Foundation, Singapore, and the Civil Aviation Authority of Singapore, under the Aviation Transformation Programme. Any opinions, findings and conclusions or recommendations expressed in this 


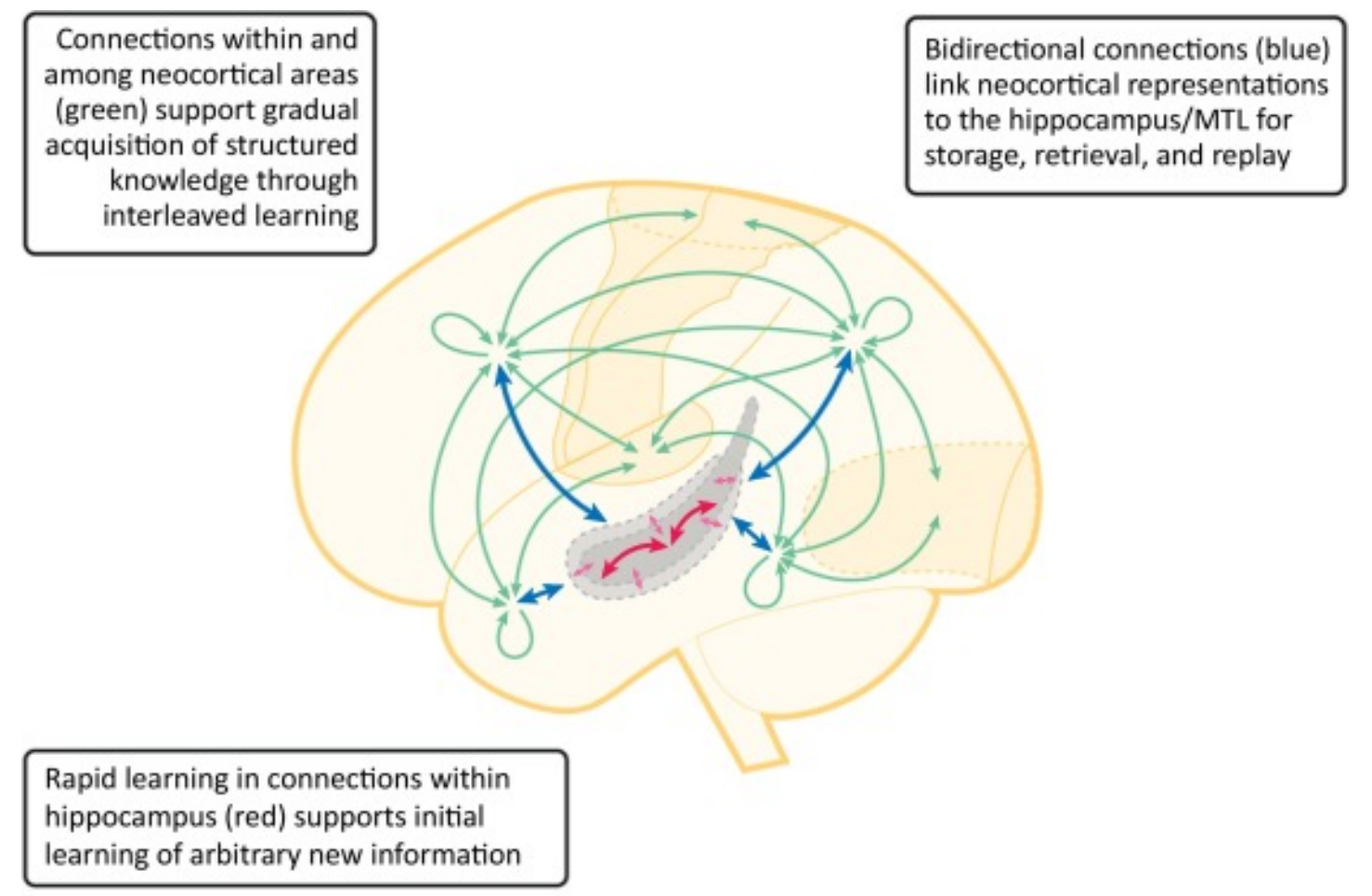

Trends in Cognitive Sciences

Figure 21: Lateral view of one hemisphere of the brain, where broken lines indicate regions deep inside the brain or on the medial surface. Primary sensory and motor cortices are shown in darker yellow. Medial temporal lobe (MTL) surrounded by broken lines, with hippocampus in dark grey and surrounding MTL cortices in light grey (size and location are approximate). Green arrows represent bidirectional connections within and between integrative neocortical association areas, and between these areas and modality specific areas (the integrative areas and their connections are more dispersed than the figure suggests). Blue arrows denote bidirectional connections between neocortical areas and the MTL. Both blue and green connections are part of the structure-sensitive neocortical learning system in the CLS theory. Red arrows within the MTL denote connections within the hippocampus, and lighter-red arrows indicate connections between the hippocampus and surrounding MTL cortices: these connections exhibit rapid synaptic plasticity (red greater than light-red arrows) crucial for the rapid binding of the elements of an event into an integrated hippocampal representation. Systems-level consolidation involves hippocampal activity during replay spreading to neocortical association areas via pathways indicated with blue arrows, thereby supporting learning within intra-neocortical connections (green arrows). Systems-level consolidation is considered complete when memory retrieval reactivation of the relevant set of neocortical representations - can occur without the hippocampus. Source: Figure 1 in [79]

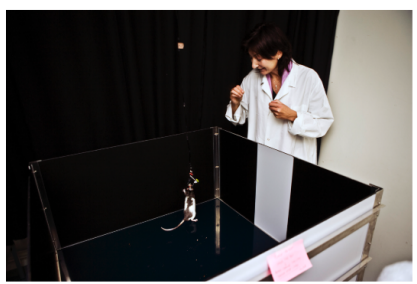

(a)

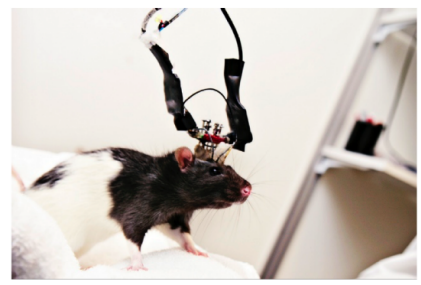

(b)

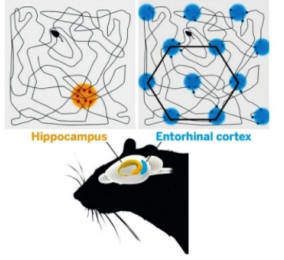

(c)

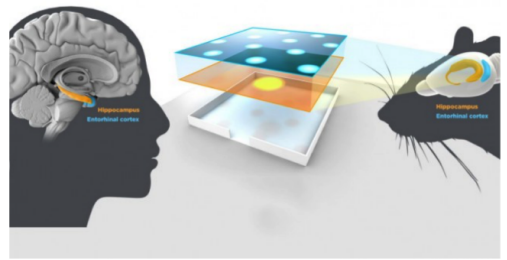

(d)

Figure 22: Place cells and grid cells. (a) The rat is moving within a square region. (b) The activity of a neuron is recorded. (c) When the rat moves around (the curve is the trajectory), each place cell fires at a particular location, but each grid cell fires at multiple locations that form a hexagon grid. (d) The place cells and grid cells exist in the brains of both rat and human. (Source of pictures: internet).

material are those of the author(s) and do not reflect the views of National Research Foundation, Singapore and the Civil Aviation Authority of Singapore. 
a

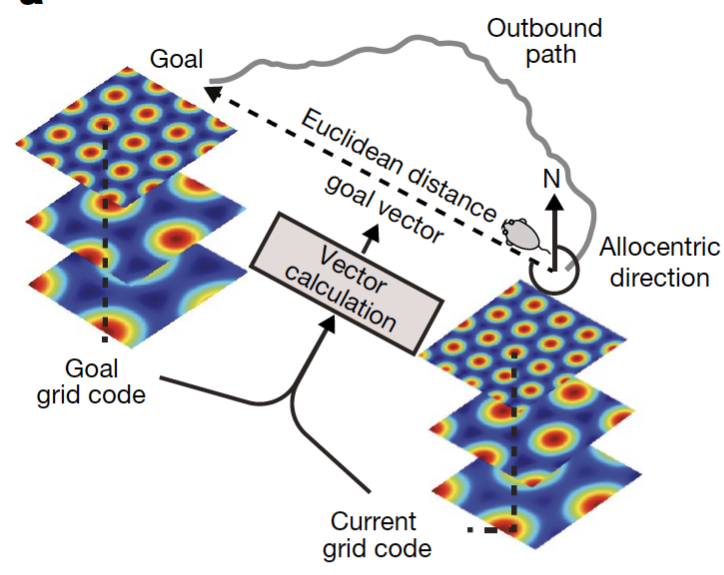

b Intra-maze cue

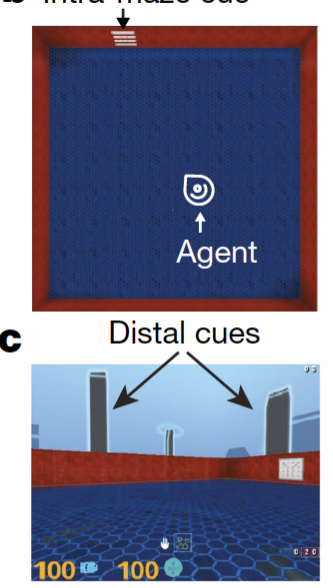

d

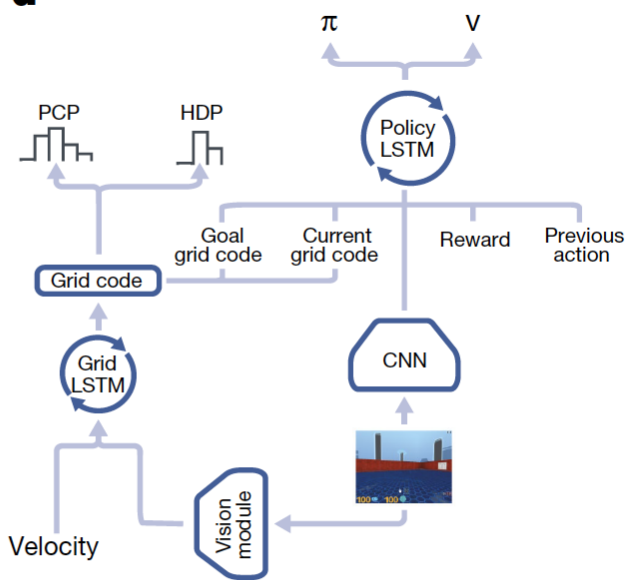

e

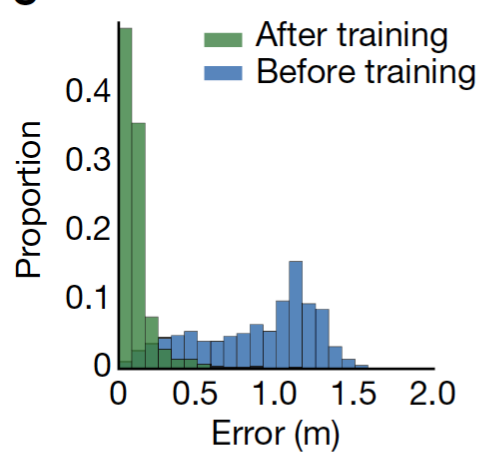

h

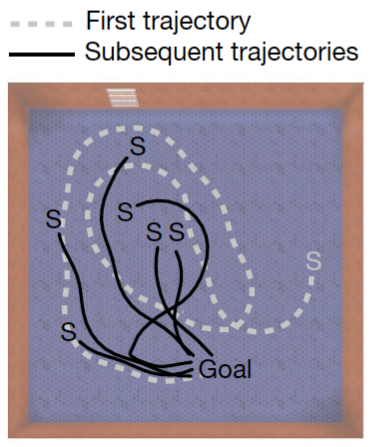

f

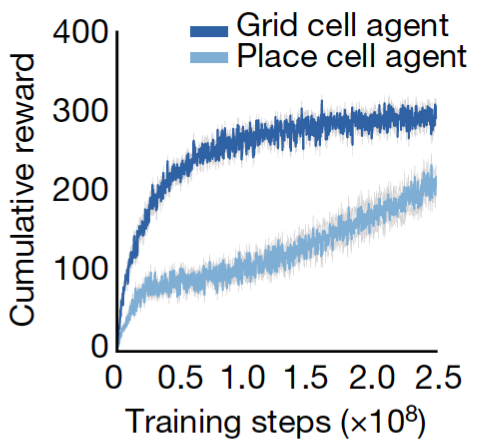

i

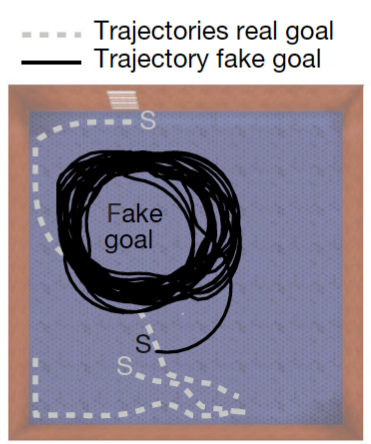

j

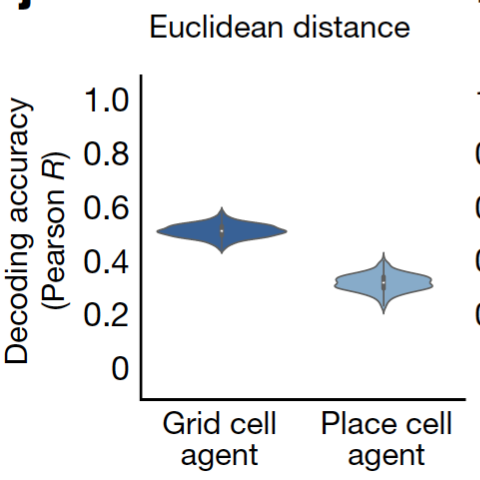

$\mathbf{g}$

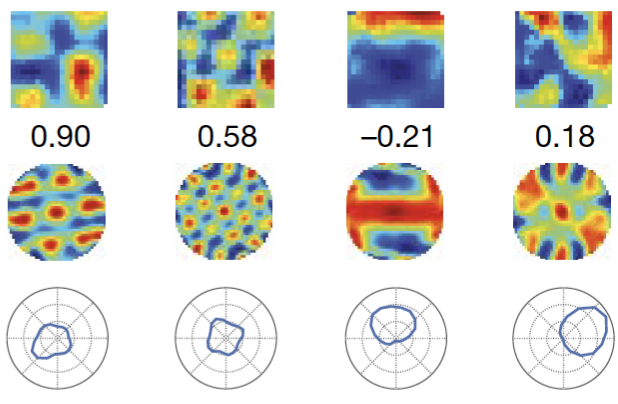

$\mathbf{k}$

Allocentric direction

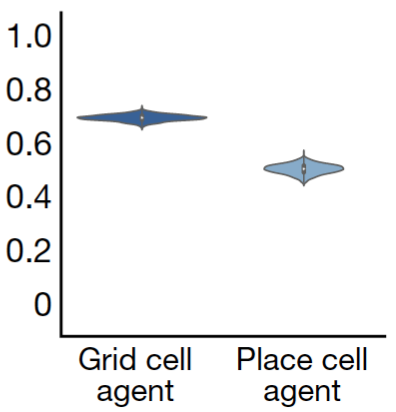

Figure 23: One-shot open field navigation to a hidden goal. a, Schematic of vector-based navigation. b, Overhead view of typical environment (icon indicates agent and facing direction). c, Agent view of b. d, Schematic of deep reinforcement learning architecture (Extended Data, Figure 5). PCP, place cell predictions; HDP, head direction cell predictions. e, Accuracy of selflocation decoded from place cell units. f, Performance of grid cell agent and place cell agent (y axis shows reward obtained within a single episode, 10 points per goal arrival, grey band displays the $68 \%$ confidence interval based on 5,000 bootstrapped samples). g, As in Figure 1, the linear layer develops spatial representations similar to entorhinal cortex. Left to right, two grid cells, one border cell, and one head direction cell. h, On the first trial of an episode, the agent explores to find the goal and subsequently navigates directly to it. 'S' denotes the starting location. i, After successful navigation, the policy LSTM was supplied with a 'fake' goal grid-code, directing the agent to this location where no goal was present. $\mathrm{j}$, $\mathrm{k}$, Decoding of goal-directed metric codes (that is, Euclidean distance and direction) from the policy LSTM of grid cell and place cell agents. The bootstrapped distribution (1,000 samples) of correlation coefficients are each displayed with a violin plot overlaid on a Tukey boxplot. Source: Figure 2 in [80] 


\section{REFERENCES}

[1] E. J. de Visser, P. J. Beatty, J. R. Estepp, S. Kohn, A. Abubshait, J. R. Fedota, and C. G. McDonald, "Learning from the slips of others: Neural correlates of trust in automated agents," Frontiers in Human Neuroscience, vol. 12, no. 309, pp. 1-15, 2018, https://doi.org/10.3389/fnhum.2018.00309.

[2] K. Drnec, A. R. Marathe, J. R. Lukos, and J. S. Metcalfe, "From trust in automation to decision neuroscience: applying cognitive neuroscience methods to understand and improve interaction decisions involved in human automation interaction," Frontiers in Human Neuroscience, vol. 10, no. 290, pp. 1-14, 2016, https://doi.org/10.3389/fnhum.2016.00290.

[3] N. Sanders, S. Choo, N. Kim, C. S. Nam, and E. P. Fitts, "Neural correlates of trust during an automated system monitoring task: Preliminary results of an effective connectivity study," in Proceedings of the Human Factors and Ergonomics Society, 2019, pp. 1-5, https://doi.org/10.1177/1071181319631409.

[4] H. A. Abbass, J. Scholz, and D. J. Reid, Eds., Foundations of trusted autonomy. Springer, 2018, retrieved from https://link.springer.com/content/pdf/ 10.1007\%2F978-3-319-64816-3.pdf.

[5] R. Parasumaran, T. B. Sheridan, and C. D. Wickens, "Situation awareness, mental workload,and trust in automation: Viable, empirically supported cognitive engineering constructs," Journal of Cognitive Engineering and Decision Making, vol. 2, no. 2, pp. 140-160, 2008, https://doi.org/10.1518/ $155534308 \mathrm{X} 284417$.

[6] K. E. Schaefer, J. Y. Chen, J. L. Szalma, and P. A. Hancock, "A meta-analysis of factors influencing the development of trust in automation: Implications for understanding autonomy in future systems," Human Factors, vol. 58, no. 3, pp. 377-400, 2016, https://doi.org/10.1177/0018720816634228.

[7] C. Kelly, M. Boardman, P. Goillau, and E. Jeannot, "Guidelines for trust in future atm systems: A literature review," EUROCONTROL, Tech. Rep. HRS/HSP-005-GUI-01, 2003, retrieved from https://www.researchgate.net/publication/311065869_Guidelines_for_Trust_in_Future_ATM_Systems_ A_Literature_Review.

[8] J. Langan-Fox, M. J. Sankey, and J. M. Canty, "Human factors measurement for future air traffic control systems," Human Factors, vol. 51, no. 5, pp. 595-637, 2009, https://doi.org/10.1177/0018720809355278.

[9] M. Madsen and S. Gregor, "Measuring human-computer trust," in 11th Australasian Conference on Information Systems, vol. 53. Australasian Association for Information Systems, 2000, pp. 6-8, retrieved from http://citeseerx.ist.psu.edu/viewdoc/download?doi=10.1.1.93.3874\&rep=rep1\&type=pdf.

[10] J. D. Lee and K. A. See, "Trust in automation and technology: Designing for appropriate reliance," Human Factors, vol. 46, no. 1, pp. 50-80, 2004, https://doi.org/10.1518/hfes.46.1.50_30392.

[11] R. Parasuraman and D. H. Manzey, "Complacency and bias in human use of automation: An attentional integration," Human Factors, vol. 52, no. 3, pp. 381-410, 2010, https://doi.org/10.1177/0018720810376055

[12] K. A. Hoff and M. Bashir, "Trust in automation: Integrating empirical evidence on factors that influence trus," Human Factors, vol. 57, no. 3, pp. 407-434, 2015, https://doi.org/10.1177/0018720814547570.

[13] R. Parasuraman, T. B. Sheridan, and C. D. Wickens, "A model for types and levels of human interaction with automation," in IEEE Transactions on Systems, Man, and Cybernetics - Part A: Systems and Humans. IEEE, 2000, pp. 286-297, retrieved from https://doi.org/10.1109/3468.844354.

[14] J. D. Lee and N. Moray, "Trust, control strategies, and allocation of function in human machine systems," Ergonomics, vol. 22, no. 10, pp. 1243-1270, 1992, https://doi.org/10.1080/00140139208967392.

[15] P. Goillau, C. Kelly, M. Boardman, and E. Jeannot, "Guidelines for trust in future atm systems: Measures," EUROCONTROL, Tech. Rep. HRS/HSP005-GUI-02, 2003, retrieved from https://www.researchgate.net/publication/311068551_Guidelines_for_Trust_in_Future_ATM_Systems_Measures.

[16] S. M. Merritt and D. R. Ilgen, "Not all trust is created equal: Dispositional and history-based trust in human-automation interactions," Human Factors, vol. 50, no. 2, pp. 194-210, 2008, https://doi.org/10.1518/001872008X288574.

[17] U. Metzger and R. Parasuraman, "The role of the air traffic controller in future air traffic management: An empirical study of active control vs. passive monitoring," Human Factors, vol. 43, no. 4, pp. 519-528, 2008, https://doi.org/10.1518/001872001775870421.

[18] D. Manzey, J. E. Bahner, and A. D. Hueper, "Misuse of automated aids in process control: Complacency, automation bias and possible training interventions," in Proceedings of the human factors and ergonomics society annual meeting, vol. 50, no. 3, 2006, pp. 220-224, retrieved from https: //doi.org/10.1177/154193120605000303.

[19] U. Metzger and R. Parasuraman, "Automation in future air traffic management: Effects of decision aid reliability on controller performance and mental workload," Human Factors, vol. 41, no. 7, pp. 35-49, 2005, https://doi.org/10.1518/0018720053653802.

[20] C. Wickens, S. Dixon, J. Goh, and B. Hammer, "Pilot dependence on imperfect diagnostic automation in simulated uav flights: An attentional visual scanning analysis," ILLINOIS UNIV AT URBANA SAVOY, Tech. Rep. AHFD-05-02, 2005, retrieved from https://apps.dtic.mil/sti/citations/ADA446167.

[21] M. T. Dzindolet, H. P. Beck, and L. G. Pierce, "Adaptive automation: Building flexibility into human-machine systems," in Understanding adaptability: A prerequisite for effective performance within complex environments, C. S. Burke, L. G. Pierce, and E. Salas, Eds. Emerald Group, 2006, pp. 213-245.

[22] J. P. Bliss, "Alarm reaction patterns by pilots as a function of reaction modality," The International Journal of Aviation Psychology, vol. 7, no. 1, pp. 1-14, 1997, http://dx.doi.org/10.1207/s15327108ijap0701_1.

[23] V. Riley, "A theory of operator reliance on automation," in Human performance in automated systems: Recent research and trends, M. Mouloua and R. Parasuraman, Eds. Lawrence Erlbaum, 1994, pp. 8-14.

[24] I. B. Ajenaghughrure, S. D. C. Sousa, and D. Lamas, "Measuring trust with psychophysiological signals:a systematic mapping study of approaches used," Multimodal Technologies and Interaction, vol. 4, no. 63, pp. 1-29, 2020, https://doi.org/10.3390/mti4030063.

[25] P. Aricò, G. Borghini, G. Di Flumeri, S. Bonelli, A. Golfetti, I. Graziani, S. Pozzi, J. P. Imbert, G. Granger, R. Benhacene, and D. Schaefer, "Human factors and neurophysiological metrics in air traffic control: a critical review," in IEEE Reviews in Biomedical Engineering. IEEE, 2017, pp. 250-263, retrieved from https://hal-enac.archives-ouvertes.fr/hal-01511343.

[26] SESAR, "Future scenarios and relevant HF concepts," SESAR, Tech. Rep. H2020-SESAR-2015-1, 2016, retrieved from http://www.stressproject.eu/ wp-content/uploads/2017/02/d1.1-future-scenarios-and-relevant-hf-concepts_v00.02.00.pdf.

[27] F. T. Eggemeier, G. F. Wilson, A. F. Kramer, and D. L. Damos, "Workload assessment in multi-task environments," in Multiple-task performance, D. L. Damos, Ed. Taylor \& Francis, 1991, pp. 207-216.

[28] P. Aricò, F. Aloise, F. Schettini, S. Salinari, D. Mattia, and F. Cincotti, "Influence of p300 latency jitter on event related potential-based brain-computer interface performance," Journal of Neural Engineering, vol. 11, no. 3, p. 035008, 2014, https://doi.org/10.1088/1741-2560/11/3/035008.

[29] G. Borghini, P. Aricò, G. Di Flumeri, G. Cartocci, A. Colosimo, S. Bonelli, A. Golfetti, J. P. Imbert, G. Granger, R. Benhacene, and S. Pozzi, "EEG-based cognitive control behaviour assessment: an ecological study with professional air traffic controllers," Scientific Reports, vol. 7, no. 1, pp. 1-16, 2017, https://doi.org/10.1038/s41598-017-00633-7.

[30] W. Klimesch, "EEG alpha and theta oscillations reflect cognitive and memory performance: A review and analysis," Brain Research Reviews, vol. 29, no. 2-3, pp. 169-195, 1999, https://doi.org/10.1016/S0165-0173(98)00056-3.

[31] G. Vecchiato, J. Toppi, A. G. Maglione, E. Olejarczyk, L. Astolfi, D. Mattia, A. Colosimo, and F. Babiloni, "Neuroelectrical correlates of trustworthiness and dominance judgments related to the observation of political candidates," Computational and Mathematical Methods in Medicine, vol. 2014, no. 434296, pp. 1-29, 2014, https://doi.org/10.1155/2014/434296.

[32] P. Aricò, G. Borghini, G. D. Flumeri, A. Colosimo, I. Graziani, J.-P. Imbert, G. Granger, R. Benhacene, M. Terenzi, S. Pozzi, and F. Babiloni, "Reliability over time of eeg-based mental workload evaluation during air traffic management (atm) tasks," in Proceedings of the IEEE Engineering in Medicine and Biology Society. IEEE, 2015, pp. 7242-7245, retrieved from https://doi.org/10.1109/EMBC.2015.7320063. 
[33] P. Aricò, G. Borghini, G. Di Flumeri, A. Colosimo, S. Bonelli, A. Golfetti, S. Pozzi, J. P. Imbert, G. Granger, R. Benhacene, and F. Babiloni, "Adaptive automation triggered by eeg-based mental workload index: A passive brain-computer interface application in realistic air traffic control environment," Frontiers in Human Neuroscience, vol. 10, no. 539, pp. 1-13, 2016, https://doi.org/10.3389/fnhum.2016.00539.

[34] T. Radüntz, N. Fürstenau, T. Mühlhausen, and B. Meffert, "Indexing mental workload during simulated air traffic control tasks by means of dual frequency head maps," Frontiers in Physiology, vol. 11, no. 300, pp. 1-13, 2020, https://doi.org/10.3389/fphys.2020.00300.

[35] K. Akash, W. L. Hu, N. Jain, and T. Reid, "A classification model for sensing human trust in machines using EEG and GSR," ACM Transactions on Interactive Intelligent Systems, vol. 8, no. 4, pp. 1-20, 2018, https://doi.org/10.1145/3132743.

[36] S. Oh, Y. Seong, S. Yi, and S. Park, "Neurological measurement of human trust inautomation using electroencephalogram," nternational Journal of Fuzzy Logic and Intelligent Systems, vol. 20, no. 4, pp. 261-271, 2020, http://doi.org/10.5391/IJFIS.2020.20.4.261.

[37] E.-S. Jung, S.-Y. Dong, and S.-Y. Lee, "Neural correlates of variations in human trust in human-like machines during non-reciprocal interactions," Scientific Reports, vol. 9, no. 9975, pp. 1-10, 2019, https://doi.org/10.1038/s41598-019-46098-8.

[38] M. Wang, A. Hussein, R. F. Rojas, K. Shafi, and H. A. Abbass, "EEG-based neural correlates of trust in human-autonomy interaction," in 2018 IEEE Symposium Series on Computational Intelligence. IEEE, 2018, pp. 350-357, https://doi.org/10.1109/SSCI.2018.8628649.

[39] S. Ogawa, T. M. Lee, A. R. Kay, and D. W. Tank, "Brain magnetic resonance imaging with contrast dependent on blood oxygenation," Proceedings of the National Academy of Sciences, USA, vol. 87, no. 24, pp. 9868-9872, 1990, https://doi.org/10.1073/pnas.87.24.9868.

[40] S. A. Huettel, A. W. Song, and G. McCarthy, Functional magnetic resonance imaging, 3rd ed. Oxford University Press, 2018.

[41] R. A. Poldrack, J. A. Mumford, and T. E. Nichols, Handbook of functional MRI data analysis. Cambridge University Press, 2018.

[42] K. Goodyear, R. Parasuraman, S. Chernyak, P. Madhavan, G. Deshpande, and F. Krueger, "Advice taking from humans and machines: An fmri and effective connectivity study," Frontiers in Human Neuroscience, vol. 10, no. 542, pp. 1-15, 2016, https://doi.org/10.3389/fnhum.2016.00542.

[43] K. Goodyear, R. Parasuraman, S. Chernyak, E. de Visser, P. Madhavan, G. Deshpande, and F. Krueger, "An fmri and effective connectivity study investigating miss errors during advice utilization from human and machine agents," Social Neuroscience, vol. 12, no. 5, pp. 570-581, 2017, https: //doi.org/10.1080/17470919.2016.1205131.

[44] K. Pushparaj, A. J. Ayeni, G. Ky, S. Alam, V. Vijayaragavan, B. Gulyás, and V. N. Duong, "A quantum-inspired model for human-automation trust in air traffic control derived from functional magnetic resonance imaging," in 9th SESAR Innovation Days, 2019, pp. 1-8, retrieved from https://www. sesarju.eu/sites/default/files/documents/sid/2019/papers/SIDs_2019_paper_55.pdf.

[45] F. Krueger, K. McCabe, J. Moll, N. Kriegeskorte, R. Zahn, M. Strenziok, A. Heinecke, and J. Grafman, "Neural correlates of trust," Proceedings of the National Academy of Sciences, USA, vol. 104, no. 50, pp. 20 084-20 089, 2007, https://doi.org/10.1073/pnas.0710103104.

[46] A. Rangel, C. Camerer, and P. R. Montague, "A framework for studying the neurobiology of value-based decision making," Nature Reviews Neuroscience, vol. 9, pp. 545-556, 2008, https://doi.org/10.1038/nrn2357.

[47] J. D. Wallis, "Cross-species studies of orbitofrontal cortex and value-based decision-making," Nature Neuroscience, vol. 15, pp. 13-19, 2012, https: //doi.org/10.1038/nn.2956.

[48] S. Russell and P. Norvig, Artificial Intelligence, 4th ed. Pearson, 2021.

[49] D. Gunning and D. Aha, "Darpa's explainable artificial intelligence (xai) program," AI Magazine, vol. 40, no. 2, pp. 44-58, 2019.

[50] A. Barredo Arrieta, N. Díaz-Rodríguez, J. Del Ser, A. Bennetot, S. Tabik, A. Barbado, S. Garcia, S. Gil-Lopez, D. Molina, R. Benjamins, R. Chatila, and F. Herrera, "Explainable Artificial Intelligence (XAI): Concepts, taxonomies, opportunities and challenges toward responsible AI," Information Fusion, vol. 58, pp. 82-115, 2020. [Online]. Available: http://www.sciencedirect.com/science/article/pii/S1566253519308103

[51] L. Spector, "Evolution of artificial intelligence," Artificial Intelligence, vol. 170, no. 18, pp. 1251-1253, 2006, special Review Issue. [Online]. Available: https://www.sciencedirect.com/science/article/pii/S0004370206000907

[52] A. M. Turing, "Computing machinery and intelligence," in Parsing the turing test. Springer, 2009, pp. 23-65.

[53] M. Haenlein and A. Kaplan, "A brief history of artificial intelligence: On the past, present, and future of artificial intelligence," California management review, vol. 61, no. 4, pp. 5-14, 2019.

[54] F.-Y. Wang, J. J. Zhang, X. Zheng, X. Wang, Y. Yuan, X. Dai, J. Zhang, and L. Yang, "Where does alphago go: From church-turing thesis to alphago thesis and beyond," IEEE/CAA Journal of Automatica Sinica, vol. 3, no. 2, pp. 113-120, 2016.

[55] M. Sadler and N. Regan, Game Changer: AlphaZero's Groundbreaking Chess Strategies and the Promise of AI. New in Chess, 2019.

[56] D. Silver, T. Hubert, J. Schrittwieser, I. Antonoglou, M. Lai, A. Guez, M. Lanctot, L. Sifre, D. Kumaran, T. Graepel, and T. Lillicrap, "A general reinforcement learning algorithm that masters chess, shogi, and Go through self-play," Science, vol. 362, no. 6419, pp. 1140-1144, 2018, https://doi.org/ $10.1126 /$ science.aar6404.

[57] Chess.com, "Alphazero crushes stockfish in new 1,000-game match," accessed: 2021-05-03. [Online]. Available: https://www.chess.com/news/view/ updated-alphazero-crushes-stockfish-in-new-1-000-game-match

[58] D. Wang, Q. Yang, A. Abdul, and B. Y. Lim, "Designing theory-driven user-centric explainable ai," in Proceedings of the 2019 CHI conference on human factors in computing systems, 2019, pp. 1-15.

[59] S. Wachter, B. Mittelstadt, and L. Floridi, "Transparent, explainable, and accountable ai for robotics," Science Robotics, vol. 2, pp. 1-5, 2017, https: //doi.org/10.1126/scirobotics.aan6080.

[60] A. B. Arrieta, N. Díaz-Rodríguez, J. Del Ser, A. Bennetot, S. Tabik, A. Barbado, S. García, S. Gil-López, D. Molina, R. Benjamins et al., "Explainable artificial intelligence (xai): Concepts, taxonomies, opportunities and challenges toward responsible ai," Information Fusion, vol. 58, pp. 82-115, 2020.

[61] C. Molnar, Interpretable Machine Learning. bookdown.org, 2019, https://christophm.github.io/interpretable-ml-book/.

[62] - Interpretable Machine Learning. bookdown.org, 2019, retrived from https://christophm.github.io/interpretable-ml-book/.

[63] N. H. Barakat and A. P. Bradley, "Rule extraction from support vector machines: A sequential covering approach," IEEE Transactions on Knowledge and Data Engineering, vol. 19, no. 6, pp. 729-741, 2007, https://doi.org/10.1109/TKDE.2007.190610.

[64] A. Chaves, M. Vellasco, and R. Tanscheit, "Fuzzy rule extraction from support vector machines," in Proceedings of the Fifth International Conference on Hybrid Intelligent Systems, Washington, DC, USA, 2005, pp. 335-340, https://doi.org/10.1109/ICHIS.2005.51.

[65] X. Fu, C. Ong, S. Keerthi, G. G. Hung, and L. Goh, "Extracting the knowledge embedded in support vector machines," in 2004 IEEE International Joint Conference on Neural Networks (IEEE Cat. No. 04CH37541), vol. 1. IEEE, 2004, pp. 291-296.

[66] A. Shrikumar, P. Greenside, and A. Kundaje, "Learning important features through propagating activation differences," in Proceedings of the 34th International Conference on Machine Learning, ser. Proceedings of Machine Learning Research, D. Precup and Y. W. Teh, Eds., vol. 70. International Convention Centre, Sydney, Australia: PMLR, 06-11 Aug 2017, pp. 3145-3153. [Online]. Available: http://proceedings.mlr.press/v70/shrikumar17a.html

[67] S. Bach, A. Binder, G. Montavon, F. Klauschen, K.-R. Müller, and W. Samek, "On pixel-wise explanations for non-linear classifier decisions by layer-wise relevance propagation," PloS one, vol. 10, no. 7, p. e0130140, 2015.

[68] I. Donadello, M. Dragoni, and C. Eccher, "Persuasive explanation of reasoning inferences on dietary data," in SEMEX: 1st Workshop on Semantic Explainability, vol. 2465. CEUR-WS. org, 2019, pp. 46-61.

[69] M. T. Keane and E. M. Kenny, "The twin-system approach as one generic solution for xai: An overview of ann-cbr twins for explaining deep learning," arXiv preprint arXiv:1905.08069, 2019.

[70] S. Lundberg and S.-I. Lee, "A unified approach to interpreting model predictions," arXiv preprint arXiv:1705.07874, 2017.

[71] L. S. Shapley, A. E. Roth et al., The Shapley value: essays in honor of Lloyd S. Shapley. Cambridge University Press, 1988. 
[72] S. Mangalathu, S.-H. Hwang, and J.-S. Jeon, "Failure mode and effects analysis of rc members based on machine-learning-based shapley additive explanations (shap) approach," Engineering Structures, vol. 219, p. 110927, 2020.

[73] SESAR, "European atm master plan: Digitalizing Europe's aviation infrastructure," 2020. [Online]. Available: https://www.sesarju.eu/masterplan

[74] F. Yang, Z. Huang, J. Scholtz, and D. L. Arendt, "How do visual explanations foster end users' appropriate trust in machine learning?" in Proceedings of the 25th International Conference on Intelligent User Interfaces, ser. IUI '20. New York, NY, USA: Association for Computing Machinery, 2020, p. 189-201. [Online]. Available: https://doi.org/10.1145/3377325.3377480

[75] S. M. Lundberg, B. Nair, M. S. Vavilala, M. Horibe, M. J. Eisses, T. Adams, D. E. Liston, D. K.-W. Low, S.-F. Newman, J. Kim, and S.-I. Lee, "Explainable machine learning predictions to help anesthesiologists prevent hypoxemia during surgery," bioRxiv, 2017. [Online]. Available: https://www.biorxiv.org/content/early/2017/10/21/206540

[76] A. Holzinger, C. Biemann, C. S. Pattichis, and D. B. Kell, "What do we need to build explainable ai systems for the medical domain?" arXiv Preprint, pp. 1-28, 2017, retrived from https://arxiv.org/abs/1712.09923.

[77] M. D. Zeiler, D. Krishnan, G. W. Taylor, and R. Fergus, "Deconvolutional networks," in IEEE Conference on Computer Vision and Pattern Recognition. IEEE, 2010, pp. 2528-2535, https://doi.org/10.1109/CVPR.2010.5539957.

[78] D. Hassabis, D. Kumaran, C. Summerfield, and M. Botvinick, "Neuroscience-inspired artificial intelligence," Neuron, vol. 95, no. 2, pp. 245-258, 2017. [Online]. Available: https://www.sciencedirect.com/science/article/pii/S0896627317305093

[79] D. Kumaran, D. Hassabis, and J. L. McClelland, "What learning systems do intelligent agents need? complementary learning systems theory updated," Trends in Cognitive Sciences, vol. 20, no. 7, pp. 512-534, 2016, https://doi.org/10.1016/j.tics.2016.05.004.

[80] A. Banino, C. Barry, B. Uria, C. Blundell, T. Lillicrap, P. Mirowski, ..., and D. Kumaran, "Vector-based navigation using grid-like representations in artificial agents," Nature, vol. 557, no. 7705, pp. 429-433, 2018, https://doi.org/10.1038/s41586-018-0102-6.

[81] Wikipedia, "Artificial neural network," accessed: 2021-03-01. [Online]. Available: https://en.wikipedia.org/wiki/Artificial_neural_network

[82] X. Chen, J. Chen, G. Cheng, and T. Gong, "Topics and trends in artificial intelligence assisted human brain research," PLoS ONE, vol. 15, no. 4, p. e0231192, 2020, https://doi.org/10.1371/journal.pone.0231192.

[83] T. Davenport and R. Kalakota, "The potential for artificial intelligence in healthcare," Future Healthcare Journal, vol. 6, no. 2, pp. 94-98, 2019, https://doi.org/10.7861/futurehosp.6-2-94.

[84] R. de Filippis, E. A. Carbone, R. Gaetano, A. Bruni, V. Pugliese, C. Segura-Garcia, and P. De Fazio, "Machine learning techniques in a structural and functional mri diagnostic approach in schizophrenia: A systematic review," Neuropsychiatric Disease and Treatment, vol. 15, pp. 1606-1627, 2019, https://doi.org/10.2147/NDT.S202418. 